\title{
Checklist of Myxomycetes from India
}

\author{
Ranade $\mathrm{VD}^{1 *}$, Korade $\mathrm{ST}^{2}$, Jagtap $\mathrm{AV}^{2}$ and Ranadive $\mathrm{KR}^{3}$ \\ ${ }^{\mathbf{1}}$ Ex- Head, Department of Botany, Abasaheb Garware College, Karve Road, Pune - 411004, Maharashtra, India \\ ${ }^{2}$ Department of Botany, University of Pune, Pune - 411007, Maharashtra, India \\ ${ }^{3}$ Waghire College, Saswad, Maharashtra, India
}

Ranade VD , Korade ST, Jagtap AV, Ranadive KR 2012 - Checklist of Myxomycetes from India Mycosphere 3(3), 358-390, Doi 10.5943 /mycosphere/3/3/9

India is rich in Myxomycetes (acellular slime moulds). They are predominantly restricted to high rainfall and humid climatic regions. Following a literature review, a checklist is provided of 373 species of Mxyomycetes, 17 varieties and 4 forms within 50 genera, 11 families and 6 orders. Most records come from the states of Himachal Pradesh, West Bengal, Uttar Pradesh, Bihar, Maharashtra, Karnataka in South India, Jammu and Kashmir.

Key Words - acellular slime moulds - fungi - literature review

\section{Article Information}

Received 24 May 2012

Accepted 30 May 2012

Published online 30 June 2012

*Corresponding author: Ranade VD - e-mail - ranade.medha@gmail.com

\section{Introduction}

Myxomycetes are plant-like in their manner of reproduction but resemble animals in the characteristics of their assimilative phase. The organism exhibits two alternating phases in its life cycle, the assimilative phase and the sporulating phase. The former consists of a free-living, acellular, mobile mass of protoplasm i.e., the plasmodium. The plasmodium absorbs nutrients from the surroundings and also engulfs solid particles including bacterial and fungal spores. The sporulating phase (sporocarp) bears spores externally on, or inside, a spore case. The spore case consists of an outer, generally persistent peridium, which envelops a free thread-like netted structure, the capilitium, which aids in spore dispersal.

Myxomycetes are a cosmopilitian group of organisms that can be found in a variety of habitats including well-manicured lawns and flower beds, and damp places, especially on old wood or other plant material undergoing decomposition. Slime molds are also common on dung and a few species may be restricted to this substratum. Another more recently recognized, specialized niche is dead branches attached to living trees.

The important taxonomic treatises available on slime moulds include Lodhi (1934), Martin \& Alexopoulos (1969), Alexopoulos (1973, 1978), Farr (1976), Thind (1977) and Lakhanpal \& Mukherji (1981).

Several workers also reported Myxomycetes from different parts of India, which were published under the banner of Fungi of India. [Martin GW (1932); Uppal BN, Patel MK \& Kamat MN (1935); Mundkur BB (1938); Patel MK, Kamat MN \& Bhide VP (1948); Ramakrishnan K, Subramanian CV (1952); Subramaniam CV, Ramakrishnan K (1956); Roy TC (1959); Vasudeva RS (1962); Tandon RN, Chandra S (1963 - 64); Mathur RS (1964); Subramaniam CV, Tyagi PD (1964), Tilak ST, Rao R (1968); Rangaswamy G, Sheshadri VS \& Lucy Channama KA (1970); Kamat MN, Patwardhan PG, Rao VG \& Sathe AV (1971); Kowalski DT (1973); Mukherji KG, Juneja RC (1974 - 75); Sarbhoy AK, Lal G \& Varshney JL (1975); Sekhon SS 


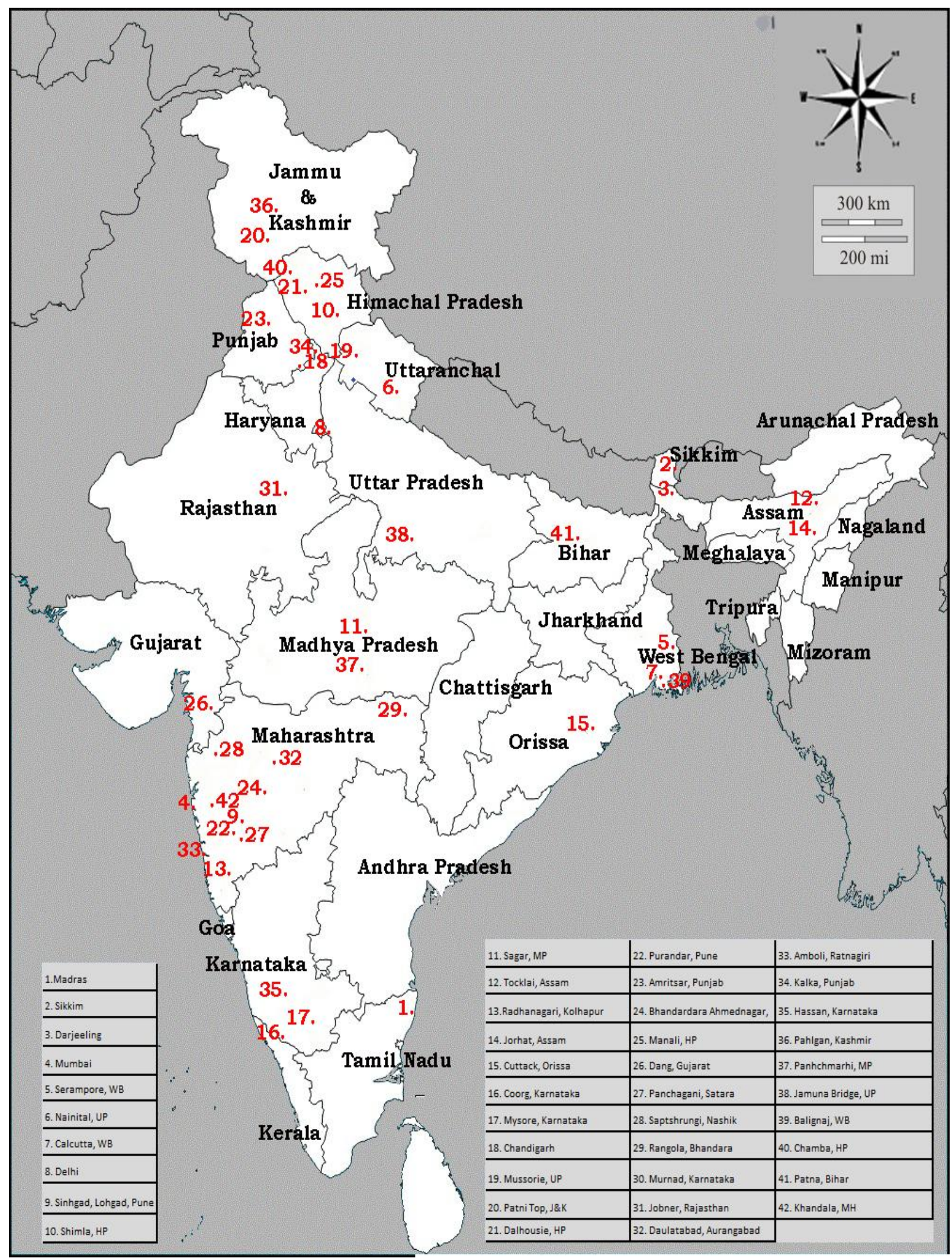

Fig. 1 - Map showing the distribution of Mxyomycetes in India

(1976 - 1979); Bilgrami KS, Jamaluddin S \& Rizwi MA (1979); Mishra RL, Ranade VD (1979); Sood R, Lakhanpal TN (1980); Sarabhoy AK, Agarwal DK \&
Varshney JL (1980 - 1986); Bhide VP, Sathe AV, Pande Alaka, Patwardhan PG \& Rao VG (1987); Hosagoudar VB, Abraham TK \& Pusphangadan P (1996); Jagtap AP, Singh NP 
(2002); Jamaluddin S, Goswami MG \& Ojha BM (2004); Tembhurne RR, Nanir SP (2011)].

\section{Indian work on Myxomycetes}

Many fungi were collected in India by Dr. Wight and these were examined by Klotzsch (1830-1833) and Berkeley (1839). Wight was perhaps the first person to collect a slime mould, Physarum cinereum (Batsch.) Pers., from Madras (Chennai), India on grass leaves in 1830 (Lakhanpal \& Mukerji, 1981).

Three species namely, Physarum conglomeratum (Fr.) Rost. Dictydiaethalium plumbeum (Schum.) Rost. and Lycogala epidendrum (L.) Fr. were collected and reported by Sir J.D. Hooker in 1849-1850 from Sikkim and Darjelling. J.C. Hobson (1862) recorded Hemitrichia serpula (Scop.) Rost. from Bombay (Mumbai). E. S. Berkley recorded 11 species of Myxomycetes between 1864 and 1882 (Lakhanpal \& Mukerji, 1981).

Pioneer work on slime moulds in India was done by Mrs. A. Drake, who collected them from different parts of India (19111927). She made 124 collections belonging to 74 species, which were then identified by Miss G. Lister. Her collection as well as of others are preserved at the Royal Botanic Garden, Kew. Lister (1924) published the first paper on Indian Myxomycetes entitled "Mycetozoa from North India". This publication was based on 36 collection made by Mrs. Drake from 19121919.

Bruhl \& Gupta (1927) published the second paper on Indian Myxomycetes describing 16 species from West Bengal.

Butler \& Bisby (1931) compiled a list of Indian Fungi and remarked, "The Myxomycetes have not been studied in India but many of these widely distributed organisms occur there". They did not include any representative of this group in their compilation. However, Lister A (1894) had already mentioned (under habit only) the occurrence of 18 species in different parts of India. Lodhi (1934) published a monograph of Indian slime moulds describing and illustrating 43 species collected by Mrs. A. Drake.

The period between 1952-1976 can be considered the Golden Period for the studies of Mxyomycetes in India. Around 1952 interest was revived in this field. Dr. K.S. Thind in North India, and Dr. V. Agnihothurudu in South India, started work on Indian Myxomycetes almost simultaneously. Thind et al. (1955-1973) in a series of 24 research papers have described about 175 species from the North Western and Eastern Himalayas and the Punjab Plains. Their contributions include 19 new species and two new varities.

Agnihothrudu and his collaborators (Agnihothrudu 1954-1956, 1965-1966; Agnihothrudu \& Chinnappa 1968-1969, Indira 1968, 1975) have described 78 species from South India, including one new species. Agnihothrudu (1958-1965) in a series of four papers described 56 species including two new species from North-East India.

Ghosh \& Dutta (1962) from Orissa, Pathak \& Ghosh (1962) from Uttar Pradesh, Kar (1964) from Calcutta, Singh, \& Pushpavathy (1965 - 1979), Singh, Pushpavathy \& Sethi (1979) from Delhi, Patwardhan \& Joshi (1975), Patil \& Ranade (1975), Ranade \& Mishra (1977, 1979), Chavan \& Kulkarni (1974), Thite (1975) from Maharashtra, Dhillon (1978), and Dhillon et al. (1978, 1979) recorded more species of Myxomycetes from their respective places.

In 1977, Prof. Thind published "Myxomycetes of India" describing and illustrating 182 species recorded up to 1973 . Lakahanpal T.N. began work on Myxomycetes of Kulu and Simla in 1965, later extending the work to other parts of Himachal Pradesh. From 1965 to 1978 he collected about one thousand specimens, including about 75 from Delhi (1969-1975). In his doctoral thesis, Lakahanpal T.N. described and illustrated 156 species. In this work he recorded 7 genera and 43 species for the first time from India and described 22 species new to science. He also reported 50 species (and one variety) from Delhi, and 83 species from Himachal Pradesh for the first time. He also amended the diagnosis of Metatrichia vesparium and Physarum laevisporum and resolved the controversy regarding the delimitation of the species of Lycogala on the basis of the ontogeny of their corticals scales. Lakhanpal \& Mukherji in a series of 19 papers entitled "Taxonomic studies on Indian Myxomycetes - 
Table 1 Myxomycetes known from India.

\begin{tabular}{|c|c|c|c|c|c|}
\hline Order & Families & Genera & Species & Variety & Forms \\
\hline 1. Ceratiomxyales & 1. Ceratiomxyaceae & 1 & 2 & 2 & - \\
\hline \multirow[t]{2}{*}{ 2. Physarales } & 2. Physaraceae & 8 & 97 & 3 & 2 \\
\hline & 3. Didymiaceae & 5 & 75 & 4 & - \\
\hline \multirow[t]{3}{*}{ 3. Liceales } & 4. Liceaceae & 2 & 29 & 1 & - \\
\hline & 5. Reticulariaceae & 4 & 14 & 1 & - \\
\hline & 6. Cribrariaceae & 3 & 21 & 3 & 2 \\
\hline \multirow[t]{2}{*}{ 4. Echinosteliales } & 7. Echinosteliaceae & 1 & 3 & - & - \\
\hline & 8. Clastodermataceae & 2 & 3 & - & - \\
\hline \multirow[t]{2}{*}{ 5. Trichiales } & 9. Dianemaceae & 3 & 3 & 1 & - \\
\hline & 10. Trichiaceae & 9 & 59 & 1 & - \\
\hline \multirow[t]{2}{*}{ 6. Stemonitales } & 11. Stemonitaceae & 12 & 67 & 1 & - \\
\hline & Total no. & 50 & 373 & 17 & 4 \\
\hline
\end{tabular}

I-XVII" and "Experimental studies on Indian Myxomycetes - I and II" described and illustrated more than 62 species from Himachal Pradesh and Delhi and have recorded their observations on the life cycle and sporangial development of some important species such as Licea scyphoides Keller \& Brookes, Macbridela cornea (G. Lister \& Cram)Alexop. and Clastoderma debaryanum Blytt. They also conducted cultural studies on some species of Didymium i.e., Didymium muscorum Lakhanpal \& Mukherji, D. karstenii Nann.Bremk., D. intermedium Schroet. and $D$. squamulosum (Alb. \& Schw.) Fr. Earlier, Thind \& Lakhanpal (1968a-d) described 16 species and Lakhanpal (1971-1973) 56 species from Himachal Pradesh, including some already described by him with Prof. Thind (loc. cit.). Kowalski \& Lakhanpal (1973) and Lakhanpal (1972) described one genus and ten species from Delhi. Lakhanpal (1972) recorded five species from Nainital in Uttar Pradesh.

Many species have been collected from natural habitats since 1976 and some more interesting forms have been obtained, in moist chambers, from the bark of living trees. The work after 1976 has seen contributions from T.N. Lakhanpal, K.G. Mukherji, S.S. Dhillon (1976-1980), S.D. Patil (1977-1979), V.D. Ranade (1977-1979), R.L. Mishra (19771979), M.L. Farr (1960-1979), H. Singh (1981), S.P. Nanir (1979-1985), S.P. Nanir et al. (1987), R.R. Tembhurne \& S.P. Nanir (2011), B.G. Rokade \& S.P. Nanir (1993), N.E. Nannenga-Bremekamp, R. Pasricha, R. Sharma, R.K. Chopra (1990), S. Kaur (1996),
Wrigley and Lado (2005).

\section{Distribution of Myxomycetes in India}

Temperature, humidity, rainfall, and topography are the main factors that determine the distribution of Myxomycetes. It is suggested that Myxomycetes attack wood after it has been partially degraded by Basidiomycetes, but their role in the decay of conifer wood may be understated.

The present study provides a checklist containing 373 species of Mxyomycetes, 17 varieties and 4 forms within 50 genera, 11 families and 6 orders. (Table 1). Liceales is the dominant order with 3 families. Stemonitaceae is the dominant family with 12 genera and Physarum is the dominant genus with up to 76 species in India. Worldwide, these 50 genera recorded in India are represented by 2344 species, 523 varieties, 4 subspecies and 58 forms (Table 2). Various classification systems of Kingdom Fungi have been proposed by mycologists, among which is the recent classification system given by Alexopoulos et al. (1996). However, the present study has taken into consideration the Ainsworth system of classification of Fungi. For classification of Mxyomycetes (also called Acellular slime moulds or True slime moulds), a system proposed by Martin et al. (1983) is used.

\section{Checklist of Mxyomycetes reported in India.}

The genera and species are listed according to the substrate and areas of India where these are recorded. 
Table 2 Distribution of Myxomycetes contributed by Indian workers in comparison with the world data.

\begin{tabular}{|c|c|c|c|c|c|c|c|c|c|c|c|c|}
\hline \multirow[t]{2}{*}{ Order and Family } & \multicolumn{4}{|c|}{ Sher Ahmad Lodhi (1934) } & \multicolumn{4}{|c|}{ Butler \& Bisby ex Vasudeva (1960) } & \multicolumn{4}{|c|}{ Agnihothrudu (1961) } \\
\hline & Gen. & sp. & var. & for. & Gen. & sp & var & for. & Gen. & sp & var & for. \\
\hline \multicolumn{13}{|l|}{ 1. Ceratiomyxales } \\
\hline \multicolumn{12}{|l|}{ 2. Physarales } & - \\
\hline Physaraceae & 6 & 20 & - & - & 7 & 26 & 1 & 1 & 6 & 48 & 1 & - \\
\hline Didymiaceae & 1 & 4 & - & - & 2 & 8 & 3 & - & 3 & 20 & 2 & - \\
\hline \multicolumn{13}{|l|}{ 3. Liceales } \\
\hline Liceaceae & - & - & - & - & - & - & - & - & - & - & - & - \\
\hline Reticulariaceae & 1 & 1 & - & - & 4 & 4 & 1 & - & 4 & 7 & 1 & - \\
\hline Cribrariaceae & 1 & 2 & - & - & 2 & 2 & 2 & - & 2 & 6 & - & - \\
\hline \multicolumn{13}{|l|}{ 4. Echinosteliales } \\
\hline Echinosteliaceae & - & - & - & - & - & - & - & - & - & - & - & - \\
\hline Clastodermataceae & - & - & - & - & - & - & - & - & - & - & - & - \\
\hline \multicolumn{13}{|l|}{ 5. Trichales } \\
\hline Dianemaceae & - & - & - & - & 1 & 1 & 1 & - & 1 & 1 & - & - \\
\hline Trichiaceae & 5 & 18 & - & - & 6 & 25 & - & - & 7 & 32 & - & - \\
\hline \multicolumn{13}{|l|}{ 6. Stemonitales } \\
\hline Stemonitaceae & 3 & 8 & - & - & 4 & 12 & - & - & 4 & 24 & 1 & - \\
\hline Total & 18 & 54 & 0 & 0 & 27 & 79 & 8 & 1 & 28 & 140 & 6 & 0 \\
\hline
\end{tabular}


Table 2 Continued Distribution of Myxomycetes contributed by Indian workers in comparison with the world data.

\begin{tabular}{|c|c|c|c|c|c|c|c|c|c|c|c|c|c|c|c|c|c|}
\hline \multirow[t]{2}{*}{ Order and Family } & \multicolumn{4}{|c|}{ Thind (1977) } & \multicolumn{4}{|c|}{ Lakhanpal \& Mukerji (1980) } & \multicolumn{4}{|c|}{ Present Study (2011) } & \multicolumn{5}{|c|}{ In World } \\
\hline & Gen. & $\mathrm{sp}$ & var & for. & Gen. & $\mathrm{sp}$ & var & for. & Gen. & $\mathrm{sp}$ & var & for. & Gen. & $\mathrm{sp}$ & var & subsp. & for. \\
\hline \multicolumn{18}{|l|}{ 1. Ceratiomyxales } \\
\hline Ceratiomyxaceae & 1 & 2 & 1 & - & 1 & 2 & 1 & - & 1 & 2 & 2 & - & 1 & 11 & 11 & - & 4 \\
\hline \multicolumn{18}{|l|}{ 2. Physarales } \\
\hline Physaraceae & 7 & 56 & - & - & 8 & 77 & 1 & 1 & 8 & 97 & 3 & 2 & 9 & 586 & 153 & 1 & 17 \\
\hline Didymiaceae & 4 & 36 & - & - & 5 & 67 & - & - & 5 & 75 & 4 & - & 7 & 361 & 44 & & 6 \\
\hline \multicolumn{18}{|l|}{ 3. Liceales } \\
\hline Liceaceae & 1 & 1 & - & - & 1 & 14 & - & - & 2 & 29 & 1 & - & 1 & 139 & 15 & - & - \\
\hline Reticulariaceae & 4 & 9 & 1 & - & 4 & 13 & - & - & 4 & 14 & 1 & & 5 & 138 & 22 & - & 1 \\
\hline Cribrariaceae & 3 & 13 & - & - & 3 & 21 & - & - & 3 & 21 & 3 & 2 & 3 & 104 & 38 & 3 & 3 \\
\hline \multicolumn{18}{|l|}{ 4. Echinosteliales } \\
\hline Echinosteliaceae & - & - & - & - & 1 & 3 & - & - & 1 & 3 & - & - & 1 & 17 & - & - & - \\
\hline Clastodermataceae & 1 & 1 & - & - & 2 & 3 & - & - & 2 & 3 & - & - & 2 & 5 & 1 & - & - \\
\hline \multicolumn{18}{|l|}{ 5. Trichales } \\
\hline Dianemaceae & 1 & 1 & - & - & 2 & 2 & - & - & 3 & 3 & 1 & - & 5 & 17 & 6 & - & - \\
\hline Trichiaceae & 7 & 29 & - & - & 7 & 44 & - & - & 9 & 59 & 1 & - & 10 & 516 & 113 & - & 18 \\
\hline \multicolumn{18}{|l|}{ 6. Stemonitales } \\
\hline Stemonitaceae & 7 & 35 & - & - & 9 & 47 & 1 & - & 12 & 67 & 1 & - & 14 & 450 & 120 & - & 9 \\
\hline Total & 36 & 183 & 2 & 0 & 43 & 293 & 3 & 1 & 50 & 373 & 17 & 4 & 58 & 2344 & 523 & 4 & 58 \\
\hline
\end{tabular}

Gen. - genus sp. - species var. - variety subsp. - subspecies for. - forms. 
Arcyodes incarnata (Alb. \& Schw.) O.F. Cook., 1902 (Trichiaceae)On dead wood, decaying twigs and bark of Diospyros melanoxylon

Kulu, Pulga, Thatchi, Mandal, Himachal Pradesh; Sagar, Madhya Pradesh.

Arcyria affinis (Rost) Nann-Brem., 1875 (Trichiaceae)

On decaying wood.

Delhi.

Arcyria assamica Agnihothrudu, 1958 (Trichiaceae)

On decaying frames of tea infected by Poria sp.

Tocklai, Assam.

Arcyria brooksii Lakhanpal \& Mukerji, 1980 (Trichiaceae)

On decaying wood of Abies pindrow.

Narkanada, Simla, Himachal Pradesh.

Arcyria cinerea (Bull.) Pers., 1801 (Trichiaceae)

On decaying wood, bark, dead stems.

Sinhgad, Pune; Sheva Islands, Bombay; Kolhapur.

\section{Arcyria denudata (L.) Wettst., 1886 (Trichiaceae) \\ On tea bark, wood, decaying frames of tea bush. \\ Cinnamara, Jorhat, Nazira, Tocklai, Assam; Cuttack, Orissa.}

Arcyria fasciculata Dhillon \& Nann.-Brem., 1978 (Trichiaceae)

On dead wood.

Uttar Pradesh.

Arcyria ferruginea Sauter., 1841 (Trichiaceae) On dead wood and decaying twigs.

Madras; Cinnamara, Jorhat, Assam; Kulu Pulga, Himachal Pradesh.

Arcyria glauca A. Lister, 1908 (Trichiaceae)

On dead wood.

Uttar Pradesh.

Arcyria globosa Schw., 1822 (Trichiaceae)
On leaves.

Lebong Forest and Rangaroon Forest, Darjeeling, West Bengal.

Arcyria gulielmae Nann. - Brem., 1971 (Trichiaceae)

On decaying wood.

Dalhousie, Himachal Pradesh.

Arcyria incarnata Pers., 1796 (Trichiaceae)

On wood and decaying bark and twigs.

Darjeeling, West Bengal; Tamilnadu; Delhi and Himachal Pradesh.

Arcyria insignis Kalchbr. \& Cooke., 1882 (Trichiaceae)

On dead stems of Saccharum officinarum.

Kolhapur, Maharashtra.

Arcyria magna Rex, 1893 (Trichiaceae)

On dead wood.

Simla, Himachal Pradesh.

Arcyria nigella Emoto., 1977 (Trichiaceae)

On bark of Cedrus deodara.

Simla, Himachal Pradesh.

Arcyria nutans (Bull.) Grev., 1824 (Trichiaceae)

Dead Wood

Simla, Himachal Pradesh.

Arcyria occidentalis (Macbr.) G. Lister, 1911 (Trichiaceae)

On dead coniferous wood.

Simla - Kufri, Himachal Pradesh.

Arcyria oerstedtii Rost., 1875 (Trichiaceae)

On dead wood.

New Delhi Ridge, India.

Arcyria rufosa S. Kaur \& Mukerji, 1996 (Trichiaceae)

On bark of Shorea robusta and Diospyros melanoxylon.

Madhya Pradesh.

Arcyria stipata (Schw.) Lister, 1894 (Trichiaceae)

On dead wood.

Simla - Kotgarh, Himachal Pradesh. 
Arcyria versicolor Phill., 1877 (Trichiaceae)

On decaying tea twigs.

Cinnamara, Assam.

Arcyria virescens Lister, 1921 (Trichiaceae)

On dead wood.

Nainital, Uttar Pradesh.

Badhamia capsulifera (Bull.) Berk., 1853

(Physaraceae)

On living mosses and bark of stumps.

Darjeeling, West Bengal.

Badhamia gracilis (Macbr.) Macbr., 1934

(Physaraceae)

Dead wood

Delhi, Karnataka

Badhamia macrocarpa (Ces.) Rost., 1874 (Physaraceae)

On dead wood, bark and twigs.

Serampore, West Bengal; Coorg, Pollibetta, Mysore, Karnataka.

Badhamia nitens Berk., 1853 (Physaraceae)

On dead wood.

Chandigarh, Himachal Pradesh.

Badhamia obovata (Peck.) F.J. Smith., 1961 (Physaraceae)

On dead wood.

Chandigarh, Himachal Pradesh.

Badhamia papaveracea Berk. \& Rav., 1873 (Physaraceae)

On dead and living exposed roots of deciduous trees and Quercus incana.

Mussoorie, Uttar Pradesh.

Badhamia utricularis (Bull.) Berk., 1852 (Physaraceae)

On dead wood and mosses.

Himachal Pradesh and Jammu \& Kashmir.

Badhamia versicolor A. Lister, 1901 (Physaraceae)

On bark, twigs and leaves of Artocarpus integrifolia.

Assam.

Badhamia viridescens Meylan, 1921
(Physaraceae)

On bark, dead twigs \& decaying leaves of Artocarpus integrifolia.

Cinnamara, Assam.

Barbeyella minutissima Meylan, 1914 (Clastodermataceae)

On decaying $\operatorname{logs}$ associated with mosses and liverworts.

Himachal Pradesh.

Brefeldia maxima (Fr.). Rostaf., 1873 (Stemonitaceae)

On dead stump.

Himachal Pradesh.

Calomyxa metallica (Berk.) Nieuwl., 1916

(Dianemaceae)

On dead forest stump.

Jamdhari Ghat, Dalhousie, Himachal Pradesh.

Calonema dissipatum Nann. - Brem., R.K. Chopra \& Lakhanpal, 1992 (Trichiaceae)

On bark of Pinus wallichiana.

Himachal Pradesh

Calonema dissipatum var. tubigranulatum Nann. - Brem., R.K. Chopra \& Lakhanpal, 1992 (Trichiaceae)

On bark of Pinus wallichiana.

Himachal Pradesh

Ceratiomyxa fruticulosa (Muller) Macbr., 1899 (Ceratiomyxaceae)

On decaying wood.

Purandar Fort, Pune, Maharashtra,

Ceratiomyxa fruticulosa var. arbuscula (Berk \& Broome) Nann. - Brem., 1975 (Ceratiomyxaceae)

On dead wood.

Dehradun, Uttar Pradesh.

Ceratiomyxa fruticulosa var. filiformis Berk. \& Br., 1869 (Ceratiomyxaceae)

In coffee plantation.

Karnataka.

Ceratiomyxa sphaerosperma Boedijn, 1927

(Ceratiomyxaceae)

On bark of Camellia sinensis (tea) infected by 
Fomes lamaensis.

Cinnamara, Assam; Balehonnur, Mysore.

Cienkowskia reticulata (Alb. \& Schw.) Rost., 1875. (Physaraceae)

On dead leaves.

Darjeeling, West Bengal.

Clastoderma debaryanum Blytt., 1880 (Clastodermataceae)

On bark of Pheonix rupicola and other trees.

Amritsar, Punjab; Simla, Himachal Pradesh; Darjeeling, West Bengal.

Clastoderma dictyosporum Lakhanpal \& Mukherji, 1976 (Clastodermataceae)

On sporophores of basidiomycetes.

Dalhousie, Himachal Pradesh.

Comatricha aequalis Peck., 1878

(Stemonitaceae)

On decaying coniferous wood.

Only in Himachal Pradesh.

Comatricha irregularis $\quad$ Rex, 1891

(Stemonitaceae)

On wooden posts, rotten stumps.

Baliganj, Calcutta, West Bengal; Mussoorie, Uttar Pradesh.

Comatricha kowalskii Lakhanpal \& Mukherji, 1977 (Stemonitaceae)

On gymnosperm wood.

Dalhousie, Himachal Pradesh.

Comatricha laxa Rost., 1874 (Stemonitaceae)

On dead moist twigs of Acacia leucophloea.

Sagar, Madhya Pradesh

Comatricha laxifila R.K. Chopra \& T.N. Lakh., 1992 (Stemonitaceae)

On bark of Pinus wallichiana.

Himachal Pradesh, India.

$\begin{array}{llll}\text { Comatricha longa } & \text { Peck., } & 1890\end{array}$

(Stemonitaceae)

On, bark, on dead and decaying bark.

Pune, Maharashtra; Mussoorie, Uttar Pradesh.

Comatricha lurida Lister, 1925 (Stemonitaceae)
On dried twig of Lantana spp.

University Campus, Delhi.

Comatricha martinii Nanir, 1983

(Stemonitaceae)

On dead wood.

Ahmadpur (Marathwada), India.

Comatricha nannengae Lakhanpal \& Mukherji, 1977 (Stemonitaceae)

On decaying gymnosperm wood covered with mosses.

Dalhousie, Himachal Pradesh.

Comatricha nigra (Pers.) Schroet., 1885 (Stemonitaceae)

On decaying wood of unidentified host and decaying frames of tea bushes infected by Poria sp., dead branches of Erythrina indica.

Jorhat, Cinnamara, Assam; Coorg, Karnataka.

Comatricha nodulifera Wollman \& Alexop., 1968 (Stemonitaceae)

On bark of Cedrus deodara.

Simla, Himachal Pradesh.

Comatricha parvispora Dhillon \& Nann.Brem., 1977 (Stemonitaceae)

On decaying trunk of wood.

Dalhousie, Uttar Pradesh.

Comatricha pulchella (Bab.) Rostaf., 1876 (Stemonitaceae)

On dead leaves of Syzygium sp., on dead wood, on decomposing tea roots, dead leaves, tea bushes infected by Ustulina zonata.

Mahabaleshwar, Amboli, Pachgani, Latur, Maharashtra; Jorhat, Nazira, Tocklai, Assam; Mussoorie, Uttar Pradesh.

Comatricha rubens Lister, 1894

(Stemonitaceae)

On dead wood.

Mussoorie, Uttar Pradesh.

Comatricha subcaespitosa Peck., 1890 (Stemonitaceae)

On decaying pieces of wood and barks.

Egmore, Madras, Tamilnadu.

Comatricha suksdorfii Ellis \& Everh., 1884 (Stemonitaceae) 
On bark of Murraya exotica.

Nandi Hills, Bangalore, Karnataka.

Comatricha tenerrima (Berk. \& M.A. Curtis.)

G. Lister, 1919 (Stemonitaceae)

On dead herbaceous twig.

Mussoorie, Uttar Pradesh; Kulu, Himachal Pradesh.

Comatricha typhoides (Bull.) Rostaf., 1873 (Stemonitaceae)

On rotting bark and wood.

Bhillaru, Khad, Mussoorie, Uttar Pradesh.

Comatricha variabilis R.K. Chopra \& T.N. Lakh., 1992 (Stemonitaceae)

On bark of Cedrus deodara.

Himachal Pradesh.

Cornuvia serpula (Wigand) Rost., 1873 (Trichiaceae)

On wood and dead leaves.

Purandar fort, Lohagad fort, Pune, Maharashtra; Darjeeling, West Bengal.

Craterium aurem (Schum.) Rostaf., 1874 (Physaraceae)

On leaves of Eleusine aegyptiaca, decaying leaves of tea; dead and decaying leaves of Quercus incana, dead and decaying twig of Barberis sp., dead and decaying leaves of Saccharum sp. and Dalbergia sissoo.

Mussoorie, Uttar Pradesh; Tocklai, Cinnamara, Nazira, Assam.

Craterium brunneum Nann. - Brem., 1973 (Physaraceae)

On dry leaf of angiosperm, on gymnosperm leaf.

Bhandardara, Ahmednagar, Maharashtra.

Craterium concinnum Rex, 1893

(Physaraceae)

On leaves.

Darjeeling, West Bengal.

Craterium costatum Dhillon \& Nann. - Brem., 1977 (Physaraceae)

On Barberis sp. leaves.

Uttar Pradesh.

Craterium leucocephalum (Pers.) Ditm., 1813
(Physaraceae)

On dead \& decaying leaves; twigs of Grevillea robusta, leaves of tea, dead leaves and branches of Quercus incana, Saccharum spp. and other plants.

Darjeeling, West Bengal; Coorg, Mysore; Hathichunghi, Assam; Mussoorie, Uttar Pradesh; Thatchi, Mandal, Himachal Pradesh.

Craterium minutum (Leers.) Fr., 1829. (Physaraceae)

On dead leaves of Grevillea robusta, dead bamboo leaves.

Mussoorie, Uttar Pradesh; Darjelling, West Bengal; Tocklai, Jorhat, Assam; Calcutta, West Bengal.

Craterium rubronodum G. List., 1915 (Physaraceae)

Habitat not given.

Serampore, Calcutta, West Bengal.

Cribraria argillacea (Pers.) Pers., 1794 (Cribrariaceae)

On dead wood.

Simla, Kotgarh, Himachal Pradesh.

Cribraria aurantiaca Schrad., 1797

(Cribrariaceae)

On decaying gymnosperm wood.

Mandal, Narkanda, Himachal Pradesh.

Cribraria costata Dhillon, Nann. - Brem., 1978 (Cribrariaceae)

On stump of conifer.

Himachal Pradesh.

Cribraria dictydiodes Cooke \& Balf., 1892 (Cribrariaceae)

On rotten wood, roots and dead mosses.

Kempty falls, Mussoorie, Uttar Pradesh.

Cribraria intricata Schrad., 1797

(Cribrariaceae)

On rotten wood, rotten roots, dead mosses etc. Mussoorie, Uttar Pradesh; Darjeeling, West Bengal; Cuttack, Orissa.

Cribraria intricata f. simlae Thind \& Lakhanpal, 1968 (Cribrariaceae)

On decaying wood. 
Mahasu, Himachal Pradesh.

Cribraria intricata Schrad. var. dictyioides (Cooke \& Balf.) List., 1894 (Cribrariaceae)

On rotten wood.

Mussoorie, Uttar Pradesh; Darjeeling, West Bengal.

Cribraria intricata var. simlae Thind \& Lakhanpal, 1968 (Cribrariaceae)

On dead wood.

Simla, Himachal Pradesh.

Cribraria languescens Rex, 1891

(Cribrariaceae)

On decaying wood.

Mussoorie, Uttar Pradesh.

Cribraria languescens f. magnigranosus

Thind \& Sohi, 1957 (Cribrariaceae)

On rotten stems and mosses.

Mussoorie, Uttar Pradesh.

Cribraria macrocarpa Schrad., 1797

(Cribrariaceae)

On dead wood.

Inner Seraj Khanag, Himachal Pradesh.

Cribraria meylanii Brandza., 1929

(Cribrariaceae)

On dead wood.

Manali, Himachal Pradesh.

Cribraria minutissima Schw., 1832

(Cribrariaceae)

On bark of living tree.

Himachal Pradesh.

Cribraria pachydictyon Nann. - Brem., 1966

(Cribrariaceae)

On bark of Pinus excelsa.

Simla, Himachal Pradesh.

Cribraria personii Nann. - Brem., 1971 (Cribrariaceae)

On decaying wood of Abies pindrow.

Simla, Himachal Pradesh.

Cribraria piriformis Schrad., 1797

(Cribrariaceae)

On decaying wood in a coniferous forest.

Dalhousie, Himachal Pradesh.
Cribraria purpurea Schrad., 1797

(Cribrariaceae)

On rotten wood.

Kulu, Pulga, Himachal Pradesh.

Cribraria rubiginosa Fries, 1829

(Cribrariaceae)

On mosses growing on a log.

Mahasu, Narkanda, Himachal Pradesh.

Cribraria splendens (Schrad.) Pers., 1801 (Cribrariaceae)

On dead wood.

Simla, Himachal Pradesh.

Cribraria tenella Schrad., 1797 (Cribrariaceae)

On rotten wood.

Simla, Kumarsain, Himachal Pradesh.

Cribraria violacea Rex, 1891 (Cribrariaceae)

On blotting paper.

University Campus, Chandigarh.

Cribrari vulgaris Schrad., 1797 (Cribrariaceae)

On dead leaves.

Bhandardara, Ahmednagar, M. S.

Cribraria vulgaris var. aurantiaca (Schrad.)

Pers., 1801 (Cribrariaceae)

On wood.

Dargeeling, West Bengal.

Diachea bulbillossa (Berk. \& Br.) Lister, 1898 (Stemonitaceae)

On attached green foliage of Hedra helix, dead leaves of Quercus incana and dead leaves and twigs of other plants.

Mussoorie, Uttar Pradesh.

Diachea khandalensis Patil \& Ranade, 1974

(Stemonitaceae)

On decaying leaves of Mangifera indica.

Khandala. Maharashtra.

Diachea leucopodia (Bull.) Rostaf., 1874 (Stemonitaceae)

On dead leaves and sticks, dead leaves of Quercus incana, dead leaves of Strobilanthus spp. leaves and culms of Eleusine aegyptia, leaves and petioles of Mangifera indica.

Mussoorie, Uttar Pradesh; Nazira, Jorhat, Assam; Cuttack, Orissa; Balihonnur, Mysore. 
Diachea megalospora Thind \& Manocha, 1964 (Stemonitaceae)

On dead ferns, dead leaves and twigs, often on living plants.

Serampore, Darjeeling - Lebong Forest, West Bengal; Mussoorie, Uttar Pradesh; Cinnamara, Assam.

Diachea radiata G. Lister \& Petch., 1916 (Stemonitaceae)

On stones, living leaves of grass and on

Boerhaavia spp.

Delhi University Campus, Delhi.

Diachea splendens $\quad$ Peck, 1878

(Stemonitaceae)

On dead leaves and twigs, on living plants.

Mussoorie, Uttar Pradesh; Dharanghati, Himachal Pradesh; Cuttack, Orissa; Delhi.

Diachea subsessilis $\quad$ Peck., 1879 (Stemonitaceae)

On dead leaves and twigs, on living herbs.

Tamilnadu; Dehradun, Uttar Pradesh; Coorg, Polibetta, Karnataka.

Diacheopsis depressa Thind \& Lakhanpal, 1969 (Cribrariaceae)

On dead leaves of Iris sp.

Kulu, Himachal Pradesh.

Dianema nivale (Meyl.) G. Lister, 1925 (Dianemaceae)

On decaying stump.

Narkanda, Himachal Pradesh.

Dictydiaethalium dictyosporum Nann. Brem., 1966 (Reticulariaceae)

Host not mentioned.

Sikkim.

Dictydiathalium plumbeum (Schum.) Rostaf., 1894 (Reticulariaceae)

On dead branches of Allophyllus cobbe, Vangueria spinosa.

Radhanagari, Kolhapur, Maharashtra.

Dictydiathalium plumbeum var., entoxanthum (Berk.) G. Lister, 1922 (Reticulariaceae)

On dead and decaying wood.

Sikkim.
Dictydium cancellatum (Batsch.) Macbr., 1899 (Cribrariaceae)

On rotten stump, bark.

Darjeeling, West Bengal; Mussoorie, Jabber, Uttar Pradesh; Jorhat, Assam; Simla, Himachal Pradesh.

Dictydium mirabile (Rost.) Meylan, 1931 (Cribrariacea)

On dead wood.

Simla, Himachal Pradesh.

Diderma alexopoulii Lakhanpal, 1978 (Didymiaceae)

On moss covered gymnosperm stump.

Himachal Pradesh.

Diderma alpino-spumarioides Thind, 1977 (Didymiaceae)

On dead leaves and twigs, sometimes on living moss.

Mussoorie - Lal Tibba, Uttar Pradesh.

Diderma alpinum (Meyl.) Meyl., 1917 (Didymiaceae)

On grass.

Pune University Campus, Pune, Maharashtra; Mussoorie, Uttar Pradesh.

Diderma asteroides (A. \& G. Lister) G. Lister, 1911 (Didymiaceae)

On decaying wood.

Simla Hills, Kotgarh, Himachal Pradesh.

Diderma badhamioides Thind,

1977

(Didymiaceae)

On mosses growing on bark of a tree.

Darjeeling, West Bengal.

Diderma chondrioderma (Deb. \& Rot.) Kuntze., 1898 (Didymiaceae)

On decaying leaves of Lagerstromia sp. and Grevillea robusta, under undetermined weeds and fronds of Pteris spp., live mosses and bark of tree, bark of Azadirechta indica, dead wood and fallen leaves.

Tocklai, Nazira, Assam; Darjeeling, West Bengal; Delhi; Thatchi, Mandal, Himachal Pradesh.

Diderma cingulatum Nann.-Brem., 1968 
(Didymiaceae)

On moist soil.

Waghai, Dang, Gujarat.

Diderma circumscissile Patil, Mishra \& Ranade, 1979 (Didymiaceae)

On dead leaves of Mangifera indica.

Lohgad, Pune, Maharashtra.

Diderma combrum Macbr. (Didymiaceae)

On dead stump

Sara, Chamba, Simla, Glen, Himachal Pradesh.

Diderma cor-rubrum Macbr., 1922

(Didymiaceae)

On moss covered stump.

Manali, Himachal Pradesh.

Diderma crustaceum Peck, 1873 (Didymiaceae)

On dead twigs and fruits of Pterocarpus marsupium, Nyctanthes arbortristis, Porana volubilis.

Madras, Tamilnadu.

Diderma darjeelingense Thind \& Sehgal, 1964 (Didymiaceae)

On leaves of herbs.

Sinchal Forest, Darjeeling, West Bengal.

Diderma deplanatum Fr., 1829 (Didymiaceae) On dead leaves and twigs of Quercus incana and other plants.

Jabber, Khet, Mussoorie, Uttar Pradesh.

Diderma effusum (Schw.) Morg., 1894 (Didymiaceae)

On rotting palms and other leaves, leaves of Setaria sp., Artocarpus integrifolia, living leaves of Cucurbitaceae, decaying leaf sheath of Areca catechu, dead leaves of Scitamineae.

Baliganj and Darjeeling, West Bengal; Tocklai, Hathi chungi, Cinnamara, Jorhat, Assam; Mussoorie, Uttar Pradesh; Madras, Tamilnadu.

Diderma globosum Pers., 1794 (Didymiaceae)

On dead leaves of Agave sp.

Kotwali Bazar, Dharamshala, Himachal Pradesh.

Diderma hemisphaericum (Bull.) Hornem.,
1829 (Didymiaceae)

On dead leaves of Eleusine aegyptia, Croton sparsiflorus, Crotolaria juncea, Salvia sp., Pleopeltis sp., Alpinia sp.

Madras, Tamilnadu.

Diderma indicum Thind \& Sehgal, 1964 (Didymiaceae)

On mosses and moss covered stump of gymnosperms.

Himachal Pradesh; West Bengal.

Diderma lohgadense Patil, Ranade \& Mishra, 1979 (Didymiaceae)

On dead and fallen leaves of Mangifera indica. Lohagad, Pune, Maharashtra.

Diderma lyallii (Massee) Macbr., 1899 (Didymiaceae)

On dead leaves.

Panchgani, Satara, Maharashtra.

Diderma marieae Patil, Mishra \& Ranade, 1979 (Didymiaceae)

On dead leaves of Mangifera indica.

Lohagad, Pune, Maharashtra.

Diderma mussooriense Thind \& Manocha, 1964 (Didymiaceae)

On dead leaves and twigs.

Jam Bridge, Mussoorie, Uttar Pradesh.

Diderma niveum (Rost.) Macbr., 1899

(Didymiaceae)

On dead leaves.

Dalhousie, Uttar Pradesh.

Diderma platycarpum var. platycarpum NannBrem., 1966 (Didymiaceae)

On dead and decaying angiosperm leaves.

Delhi.

Diderma punense Patil, Ranade \& Mishra, 1979 (Didymiaceae)

On dead leaves.

Pune, Maharashtra.

Diderma radiatum (L.) Morg., 1894

(Didymiaceae)

On dead wood.

Bhimtal, Uttar Pradesh; Kulu, Himachal 
Pradesh.

Diderma radiatum (L.) Morg. var. umbilicatum (Pers.) G. Lister, 1913 (Didymiaceae)

On dead wood.

North India.

Diderma reticulosporum Nann.-Brem., Mukherji \& Pasricha, 1984 (Didymiaceae)

On dead leaf.

Madhya Pradesh, India.

Diderma rimosum U. Eliass and Nann.-Brem., 1983 (Didymiaceae)

On dry leaves of angiosperm.

Rengola, Lakhandur, Bhandara; Saptashrungi, Nashik.

Diderma roanense (Rex) Macbr., 1899 (Didymiaceae)

On dead wood.

Manali, Kulu, Himachal Pradesh.

Diderma rugosum (Rex) Macbr., 1899 (Didymiaceae)

On decomposing leaves of Cocos nucifera Agriculture Horticulture Garden, Madras, Tamil Nadu.

Diderma simplex (Schroet.) G. Lister, 1911 (Didymiaceae)

On dead leaves.

Darjeeling, West Bengal.

Diderma spumariodes (Fr. \& Palmquist) Fr., 1829 (Didymiaceae)

On living moss and dead leaves of Quercus incana.

Mussoorie, Uttar Pradesh.

Diderma subdictyospermum (Rost.) List., 1911 (Didymiaceae)

On fern fronds, dead wood.

Kankeshwar, Maharashtra; Kangra, Himachal Pradesh; Mysore, Karnataka; Thatchi, Mandal, Himcahal Pradesh.

Diderma testaceum (Schrad.) Pers., 1801 (Didymiaceae)

On dead leaves of Agave sp and Tectona grandis, on dead leaf in Cardamom plantation.
Mahabaleshwar, Pachgani, Amboli, Maharashtra; Murnad, Coorg, Mysore, Karnataka.

Diderma trevelyani (Grev.) Fries, 1829

(Didymiaceae)

On dead wood.

Himachal Pradesh.

Didymium anellus Morg., 1894 (Didymiaceae)

On bark of Mangifera indica.

Dehradun, Uttar Pradesh.

Didymium chrysoporum Lakhanpal \& Mukerji, 1979 (Didymiaceae)

On decaying angiosperm leaves,

Delhi.

Didymium clavus (Alb. \& Schw.) Rab., 1844 (Didymiaceae)

On decaying bamboo mats, palm leaves, twigs of Ipomea sp.

Madras, Tamil Nadu.

Didymium crustaceum $\quad$ Fr., 1829 (Didymiaceae)

On decaying bamboo mat, dead twig of Ipomea sp., living herbaceous twigs.

Madras, Tamilnadu; Kulu, Himachal Pradesh.

Didymium delhianum Lakhanpal \& Mukerji, 1979 (Didymiaceae)

On decaying angiosperm leaves.

Delhi.

Didymium difforme (Pers.) S.F. Gray, 1821 (Didymiaceae)

On dead leaves of Tectona grandis, on dead and decaying leaves and twigs of Helianthus annuus and other members of Compositae.

Manabaleshwar, Katraj, Bhimashankar, Maharashtra; Mussoorie, Uttar Pradesh.

Didymium disciforme Kowaleski \& Lakhanpal, 1973 (Didymiaceae)

On dead leaves of Ficus benghalensis.

Delhi.

Didymium discoideum Thind \& Sehgal, 1964

(Didymiaceae)

On dead leaves.

Senchal Forest, Darjeeling, West Bengal. 
Didymium dubium Rostaf., 1874 (Didymiaceae)

On dead palm leaves, twigs and bamboo mats, on grass and ferns.

Madras, Tamilnadu; Kulu, Himachal Pradesh.

Didymium flexuosum Yamashiro, 1936 (Didymiaceae)

On dead leaves, leaf litter in coffee plantations. Nandi hills, Madal, Himachal Pradesh; Polibetta, Coorg, Karnataka.

Didymium floccosum Martin, Thind \& Rehill, 1959 (Didymiaceae)

On dead leaves.

Dehradun, Uttar Pradesh.

Didymium fulvum Sturgis, 1917

(Didymiaceae)

On decaying wood.

Haretta, Hamirpur, Himachal Pradesh.

Didymium haretianum Lakhanpal \& Mukerji, 1979 (Didymiaceae)

On decaying angiosperm leaves.

Hamirpur, Himachal Pradesh.

Didymium intermedium Schroet., 1896

(Didymiaceae)

On bark.

Delhi.

Didymium iridis (Ditm.) Fr., 1829 (Didymiaceae)

On dead leaves of Quercus incana, living leaves of Gerbera sp., on twigs of Diospyros melanoxylon.

Kulu, Himachal Pradesh; Mussoorie, Uttar Pradesh; Sagar, Madhya Pradesh.

Didymium karstensii Nann.-Brem., 1964 (Didymiaceae)

On decaying leaves of Ficus religiosa.

Delhi.

Didymium lenticulare Thind \& Lakhanpal, 1969 (Didymiaceae)

On fern fronds.

Kulu Hills, Himachal Pradesh.

Didymium leoninum Berk. \& Br., 1875
(Didymiaceae)

On dead leaves of Mahonia sp., ferns and other plants.

Mussoorie, Uttar Pradesh.

Didymium megalosporum Berk. \& Curt., 1873

(Didymiaceae)

On litter of Coffea arabica.

Coorg, Karnataka; Madras, Tamil Nadu.

Didymium melanospermum (Pers.) Macbr., 1899 (Didymiaceae)

On dead leaves and twigs.

Tamil Nadu; Uttar Pradesh.

Didymium melanospermum Macbr. var. bicolor List., (Didymiaceae)

On dead wood and leaves.

Landour, Mussoorie, Uttar Pradesh; Jobner, Rajasthan.

Didymium minus (Lister) Morg., 1894 (Didymiaceae)

On dead leaves and twigs, sometimes on living grasses.

Palni Hills, Tamilnadu; Mussoorie, Uttar Pradesh; Darjeeling, West Bengal.

Didymium mirabile (Rost.) Meylan (Didymiaceae)

On dead wood.

Simla, Baghi, Himachal Pradesh.

Didymium muscorum Lakhanpal \& Mukerji, 1976 (Didymiaceae)

On decaying leaves and twigs.

Delhi.

Didymium nigripes (Link) Fr., 1818 (Didymiaceae)

On dead leaves, vegetable debris.

Madras, Tamil Nadu; Serampore, Calcutta, West Bengal; Delhi; Assam; Mussoorie, Uttar Pradesh.

Didymium nigripes (Link) Fr. var. xanthopus (Ditm.) List., 1894 (Didymiaceae)

On decaying leaves.

Serampore, West Bengal.

Didymium nigrisporum Nann.-Brem., K.G. Mukherji \& R. Pasricha, 1984 
(Didymiaceae)

On herbaceous stem.

Madhya Pradesh.

Didymium ochroideum G. List., 1931

(Didymiaceae)

On dead leaves.

Serampore, West Bengal.

Didymium ovoideum Nann.-Brem., 1958 (Didymiaceae)

On dead leaves.

Baghi, Himachal Pradesh.

Didymium perforatum Yamashiro, 1936

(Didymiaceae)

On living grass leaves.

Chandigarh.

Didymium projectile Lakhanpal \& Mukerji, 1979 (Didymiaceae)

On living fern fronds, dry needles of Cedrus deodara.

Himachal Pradesh.

Didymium quitense (Pat.) Torrend, 1908

(Didymiaceae)

On grass stem, dead potato leaves.

Dalhousie, Himachal Pradesh.

Didymium saturnus Keller, 1970

(Didymiaceae)

On cotton and maize leaves, kangaroo dung.

Delhi.

Didymium simlense Lakhanpal \& Mukerji, 1979 (Didymiaceae)

On dead leaves of Quercus incana.

Himachal Pradesh.

Didymium squamulosum (Alb. \& Schw.) Fr. \& Palmquist, 1818 (Didymiaceae)

On dead leaves of Quercus incana, twigs, bark and other substrates, decaying leaves of Diospyros melanoxylon.

Mussoorie Uttar Pradesh; Delhi; Sagar, Madhya Pradesh.

Didymium sturgisii Hagelst., 1937 (Didymiaceae)

On Pinus sp. bark.
Tamil Nadu; Uttar Pradesh.

Didymium tubi-crystallinum Nann.-Brem. \& Critchfield, 1988 (Didymiaceae)

On dead herbaceous twig.

Milind Garden, Aurangabad, Maharashtra.

Didymium vaccinum (Dur. \& Mont.) Buchet, 1920 (Didymiaceae)

On rotten unidentified leaves.

Cuttack, Orissa.

Didymium verrucosporum Weldem., 1954 (Didymiaceae)

On decaying stem of Euphorbia royleana and Saccharum sp., leaf litter.

Kulu, Himachal Pradesh; Madras, Tamil Nadu.

Echinostelium cribrariodes Alexop., 1961

(Echinosteliaceae)

On bark of Rhododendron sp.

Simla, Himachal Pradesh.

Echinostelium elachiston Alexop., 1958 (Echinosteliaceae)

On bark of Eucalyptus sp.

Delhi University; Shevan Islands, Bombay.

Echinostelium minutum de Bary, 1873 (Echinosteliaceae)

On bark of Populus sp.

Simla, Himachal Pradesh; Shevan Islands, Bombay; Jammu \& Kashmir.

Elaeomyxa miyazakiensis (Emoto) Hagelstein, 1942 (Stemonitaceae)

On rotten wood.

Cuttack, Orissa.

Erionema aureum Penzig, 1898

(Physaraceae).

On moss covering decaying wood of Abies pindrow.

Narkanda, Simla, Himachal Pradesh.

Fuligo cinerea (Schw.) Morg., 1896 (Physaraceae)

On bark and cow dung, on dead leaves of Dalbergia sissoo, Saccharum sp. and on rotten cloth pieces.

S. P. College campus, Pune, Maharashtra; 
Doiwala, Dehradun, Uttar Pradesh; Delhi.

Fuligo intermedia Macbr., 1922 (Physaraceae) On dry stem of Euphorbia sp.

Daulatabad, Aurangabad, Maharashtra.

Fuligo megaspora Sturgis, 1913 (Physaraceae)

On wood, soil and leaves.

Chandigarh, Himachal Pradesh.

Fuligo septica for. ovata (Schaeff.) Pers., (Physaraceae)

On decaying bark, leaves.

Assam; Chandigarh, Himachal Pradesh; Tamil Nadu.

Fuligo septica (L.) F.H. Wigg, 1780 (Physaraceae)

On decaying vegetable debris in pigeon pea field.

Agricultural Horticultural Gardens, Madras, Tamil Nadu.

Hemitrichia calyculata (Speg.) Farr, 1974 (Trichiaceae)

On wood, on decaying gymnosperm wood.

Kanakeshwar, Mahabaleshwar, Maharashtra; Assam; Mandi, Narkanda, Himachal Pradesh; Orissa, Uttar Pradesh.

Hemitrichia clavata (Pers.) Rostaf., 1873 (Trichiaceae)

On wood, on dead wood and rotten wood.

Mahabaleshwar, Maharashtra; Darjeeling, West Bengal; Mussoorie, Uttar Pradesh; Madal, Kulu, Manali, Himachal Pradesh; Sagar, Madhya Pradesh.

Hemitrichia imperalis G. Lister, 1929 (Trichiaceae)

On dead and decaying wood.

Kulu, Himachal Pradesh.

Hemitrichia karstenii (Rostaf.) List., 1894 (Trichiaceae)

On dead wood and palm.

Darjeeling, Baliganj, Calcutta, West Bengal; Assam.

Hemitrichia leotrichia (Lister) G. Lister, 1911 (Trichiaceae)
On Acacia pinnata, on bark of Pinus excelsa kept in moist chamber.

Amboli, Ratnagiri, Maharashtra; Himachal Pradesh.

Hemitrichia serpula (Scop.) Rostaf., 1873 (Trichiaceae)

On decaying leaves of palm like Areca catechu, Cocos nucifera, Agave sp., on dead wood, mosses, leaf blade of Musa paradisiaca. Mahabaleshwar, Purandar fort, Vani, Amboli, Maharashtra; Darjeeling, West Bengal; Mussoorie, Uttar Pradesh; Tocklai, Assam; Panchmarhi, Madhya Pradesh.

Hemitrichia stipitata (Massee) Macbr., 1899 (Trichiaceae)

On dead wood, living mosses, decaying wood of Albizia procera, bark of Mangifera indica, root and root bark of Tea bush.

Mussoorie, Uttar Pradesh; Tocklai, Jorhat, Assam; Polibetta, Coorg, Karnataka; Cuttack, Orissa; Thatchi, Mandi, Himachal Pradesh.

Hemitrichia vesparium (Batsch) Macbr., 1899 (Trichiaceae)

On dead wood, rotten wood.

Kotgarh, Simla, Himachal Pradesh; Mussoorie, Uttar Pradesh; Balehonnur, Mysore, Karnataka.

Lamproderma alexopouli Lakhanpal \& Mukerji, 1979 (Stemonitaceae)

On decaying angiosperm leaves and herbaceous twigs.

Delhi.

Lamproderma arcyrionema Rost., 1874. (Stemonitaceae)

On dead leaves, wood and mosses.

Mussoorie, Uttar Pradesh; Darjeeling, West Bengal; Simla, Himachal Pradesh; Kalka, Punjab.

Lamproderma collinsi Lakhanpal \& Mukherji, 1979 (Stemonitaceae)

On decaying wood of Abies pindrow. Narkanda, Simla, Himachal Pradesh. Lamproderma columbinum (Pers.) Rostaf., 1873 (Stemonitaceae)

On moss covered stump.

Kulu, Pulja, Himachal Pradesh. 
Lamproderma echinulatum (Berk.) Rostaf., 1876 (Stemonitaceae)

On moss.

Darjeeling, West Bengal.

Lamproderma griseum Thind \& Lakhanpal, 1969 (Stemonitaceae)

On decaying leaves of Iris sp.

Kulu, Manali, Himachal Pradesh.

Lamproderma retispora Dhillon \& Nann. Brem., 1977 (Stemonitaceae)

On Quercus sp. leaves.

Uttar Pradesh.

Lamproderma scintillans (Berk. \& Br.) Morgan, 1894 (Stemonitaceae)

On dead leaves of Mangifera indica and Tectona grandis, on decaying palm leaves.

Sinhgad, Lohagad, Pachgani, Pune, Khandala, Maharashtra; Darjeeling, West Bengal; Mussoorie, Baliganj, Uttar Pradesh.

Lamproderma thindianum Lakhanpal \& Mukerji, 1979 (Stemonitaceae)

On decaying leaves of Iris sp.

Kulu, Manali, Himachal Pradesh.

Lamproderma verrucosum Martin, Thind \& Sohi, 1957 (Stemonitaceae)

On dead leaves of Quercus incana.

The Company Garden, Mussoorie, Uttar Pradesh.

Leocarpus fragilis (Dicks.) Rostaf., 1875 (Physaraceae)

On bark of Cedrus deodara, dead needles of Pinus sp. and Cedrus sp.

Leudon, Mussoorie, Uttar Pradesh; Simla, Himachal Pradesh.

Lepidoderma effusum Rokade \& Nanir, 2004 (Didymiaceae)

On dry straw of angiosperm plants.

Kolegaon, Malsiras, Solapur, Maharashtra; Gujarat; Madhya Pradesh.

Lepidoderma tigrinum (Schrad) Rostaf., 1873 (Didymiaceae)

On dead wood, fallen needles of Pinus sp. and Cedrus sp.
Kodai forest, Karnataka; Mussoorie, Uttar Pradesh; Kulu, Himachal Pradesh.

Lepidodermopsis leonina (Berk. \& Br.) Hohn., 1909 (Didymiaceae)

On dead and decaying leaves, fern fronds.

Simla, Himachal Pradesh; Uttar Pradesh.

Lepidodermopsis martinii T. N. Lakh., 1978 (Didymiaceae)

On stumps of Cedrus deodara.

Himachal Pradesh.

Leptoderma irridescens G. Lister, 1913 (Stemonitaceae)

On decaying gymnosperm wood.

Kalatop, Dalhousie, Himachal Pradesh; Tamil Nadu.

Licea belmontiana Nann.-Brem., 1966 (Liceaceae)

On bark of Abies pindrow, Pinus sp.

Himachal Pradesh; Shivan Islands, Bombay.

Licea biforis Morgan., 1893 (Liceaceae)

On decaying wood.

Darjeeling, West Bengal; Chandigarh, Himachal Pradesh.

Licea castanea G. Lister., 1911 (Liceaceae)

On bark of Aesculus indica.

Chandigarh, Himachal Pradesh.

Licea confundens T.N. Lakhanpal, N.E. Nann.-Brem. \& R.K. Chopra, 1990 (Liceaceae) On bark of Quercus incana.

Himachal Pradesh.

Licea elloriana Nanir \& Rokade, 2003 (Liceaceae)

On dry leaves of angiosperm.

Panhala, Kolhapur, Maharashtra; Gujarat, Madhya Pradesh.

Licea erecta Thind \& Dhillon, 1967 (Liceaceae)

On decaying bamboo twig.

Darjeeling, West Bengal.

Licea exiguum (Authority and year not mentioned) (Liceaceae) 
On unidentified bark.

Sagar, Madhya Pradesh.

Licea floriformis T.N. Lakhanpal \& R.K. Chopra, 1990 (Liceaceae)

On bark of Quercus incana.

Himachal Pradesh.

Licea hydragyra Nann.-Brem., T.N. Lakhanpal \& R.K. Chopra, 1990 (Liceaceae)

On bark of Quercus incana.

Himachal Pradesh.

Licea lilacina Nann.-Brem., T.N. Lakhanpal \& R.K. Chopra, 1990 (Liceaceae)

On bark of Quercus incanca.

Himachal Pradesh.

Licea kleistobolus Martin, 1942 (Liceaceae)

On bark.

Shivan Islands, Bombay.

Licea marginata Nann.-Brem., 1965

(Liceaceae)

On fallen leaves of Tectona grandis.

Nandoshi, Vitthalwadi, Pune, Maharashtra.

Licea minima Fr., 1829 (Liceaceae)

On decaying gymnosperm wood.

Glen, Simla, Himachal Pradesh.

Licea morchelloides T.N. Lakhanpal, Nann.Brem. \& R.K. Chopra, 1990 (Liceaceae)

On bark of Quercus incana.

Himachal Pradesh.

Licea mysorensis Agnihothrudu, 1965

(Liceaceae)

On bark of Coffea arabica.

Saklespur, Hassan, Karnataka.

Licea nigromarginata Nann.-Brem., T.N. Lakhanpal \& R.K. Chopra, 1990 (Liceaceae)

On bark of Pinus wallichiana.

Himachal Pradesh.

Licea operculata (Wingate) Martin., 1942 (Liceaceae)

On bark of Cedrus deodara.

Simla, Himachal Pradesh.
Licea pedicellata (Gilbert.) Gilbert, 1942 (Liceaceae)

On bark of Butea monosperma and Acacia leucophloea, Pinus excelsa.

Sagar, Madhya Pradesh; Simla, Himachal Pradesh.

Licea perexigua Brookes \& Keller, 1977 (Liceaceae)

On bark of living tree.

Simla, Himachal Pradesh.

Licea punctiformis Martin, 1969 (Liceaceae)

On horse dung.

Nainital, Uttar Pradesh.

Licea scyphoides Brookes \& Keller., 1977. (Liceaceae)

On bark of living trees.

Simla, Himachal Pradesh.

Licea sinhgadensis Patil, Ranade \& Mishra., 1977 (Liceaceae)

On dead leaves of Tectona grandis.

Sinhgad, Pune, Maharashtra.

Licea tenera Jahn, 1919 (Liceaceae)

On horse dung.

Delhi; Chandigarh, Himachal Pradesh.

Licea testudinacea Nann - Brem., 1965 (Liceaceae)

On bark of Abies pindrow.

Himachal Pradesh.

Licea tuberculata var. papillata Nann.-Brem., T.N. Lakhanpal \& R.K. Chopra, 1990 (Liceaceae)

On bark of Quercus incana.

Himachal Pradesh.

Licea variabilis Schrad, 1797 (Liceaceae)

On horse dung.

Delhi.

Licea verrucospora (T.N. Lakh., Nann.-Brem. \& R.K. Chopra) D. Wrigley \& Lado, 2005 (Liceaceae)

On bark of living Quercus incana.

Simla, Himachal Pradesh. 
Lindbladia tubulina Fr., 1849 (Cribrariaceae)

On decaying gymnosperm wood.

Simla, Narkanda. Himachal Pradesh.

Lycogala conicum Pers., 1801

(Reticulariaceae)

On dead wood, fresh stump of Pinus roxburghii.

Mussoorie, Uttar Pradesh; Manali, Mandal, Thatchi, Simla, Jakhu \& Ellen, Himachal Pradesh.

Lycogala epidendrum (L.) Fries, 1829 (Reticulariaceae)

On dung, decaying leaves, dead wood

Mahabaleshwar, Pachgani, Vani hills, Maharashtra; Sikkim; Darjeeling, West Bengal; Nilgiri hills, Madras; Pahlgan, Kashmir.

Lycogala exiguum Morgan, 1893 (Reticulariaceae)

On rotten wood, dead stump of Cedrus sp.

Saklespur, Karnataka; Assam; Himachal Pradesh.

Lycogala flavofuscum (Ehrenb.) Rost., 1873 (Reticulariaceae)

On dead gymnospermous wood.

Nainital, Uttar Pradesh.

Lycogala mysorensis (Agnihothrudu) Agnihothrudu, 1966 (Reticulariaceae)

On soil.

Ossoor Coffee Estate, Karnataka.

Macbrideola coprophila Nann.-Brem., Mukerji and N. Singh, 1979 (Stemonitaceae)

On dung of peacock.

Rajashthan; Delhi.

Macbrideola cornea (G. Lister \& Cran) Alexo., 1967 (Stemonitaceae)

On bark of Abies sp. and Pinus excelsa.

Jaku hills, Narkanda, Simla, Himachal Pradesh.

Macbrideola indica (Sarbhoy, S.M. Singh \& D.K. Agarwal) Nann.-Brem., Lakhanpal, Mukerji \& H. Singh, 1981 (Stemonitaceae)

On dung of peacock.

Rajasthan; Delhi.
Macbrideola robusta Pal \& Mukherji, 1977 (Stemonitaceae)

On bark of Rhododendron sp.

Simla, Himachal Pradesh.

Margarita mettalica (Berk.) List., 1894

(Dianemaceae)

On dead wood

Kotgarh, Simla hills, Himachal Pradesh.

Margarita mettalica (Berk.) List. var. plasmodiocarpa (Blytt) R.E. Fries., 1925

(Dianemaceae)

On dead wood.

Western Himalayas.

Metatrichia arundinariae (Rammeloe) Lakhanpal \& Mukherji, 1977 (Trichiaceae)

On decaying gymnosperm wood.

Himachal Pradesh.

Metatrichia vesparium (Batsch.) Nann.Bremk. ex. G.W. Martin \& Alexop., 1969 (Trichiaceae)

On dead and rotten wood of Poria sp.

Simla, Kotgarh, Himachal Pradesh; Mussoorie, Jabbar khet, Uttar Pradesh.

Oligonema flavidum (Peck) Peck, 1879 (Trichiaceae)

On dead leaves.

Kotgarh, Simla hills, Himachal Pradesh; Serampore, Darjeeling, West Bengal; Mussoorie, Uttar Pradesh.

Perichaena chrysosperma (Currey) List., 1894 (Trichiaceae)

On dead wood, dead and decaying leaves of Agave sp.

Darjeeling, West Bengal; Mussoorie, Uttar Pradesh; Tamil Nadu.

Perichaena corticalis (Batsch.) Rost., 1875 (Trichiaceae)

On bark.

Chandigarh.

Perichaena depressa Libert, 1837

(Trichiaceae)

On bark of Aleuria montana, dead wood of a 
deciduous tree and bark of a tree. Darjeeling, West Bengal; Tocklai, Assam; Dehradun, Uttar Pradesh; Delhi; Tamil Nadu; Himachal Pradesh; Amritsar, Punjab.

Perichaena microspora Penz. \& A. Lister, 1898 (Trichiaceae)

On dry angiosperm leaves.

Bada Mahadev, Panchmarhi, Madhya Pradesh

Perichaena minor (G. Lister) Hageslt., 1943 (Trichiaceae)

On dead bark of Tamarindus indica.

Sagar, Madhya Pradesh.

Perichaena quadrata Macbr., 1899

(Trichiaceae)

On wood.

Darjeeling, West Bengal.

Perichaena thindii Nanir, 1981(Trichiaceae)

On dead wood.

Daulatabad, Aurangabad, Maharashtra.

Perichaena vermicularis (Schw.) Rost., 1876 (Trichiaceae)

On decomposing leaves of Bignonia unguiscati, decaying balls of Gossypium arboretum, stem galls of Acacia leucophloea.

Madras, Tamilnadu; Tocklai, Assam; Dehradun, Uttar Pradesh; New Delhi.

Physarella oblonga (Berk. \& Curt.) Morg., 1896 (Physaraceae)

On decaying leaves of Ficus sp., on decaying wood and polypore, soil, bark of tea bushes, decaying bark of Albizia stipulate.

Pune Universtiy Campus, Pratapgad, Mahabaleshwar, Maharashtra; Darjeeling, West Bengal; Dehradun, Uttar Pradesh; Jorhat, Assam; Cuttack, Orissa.

Physarella oblonga f. alba Alexop., 1964 (Physaraceae)

On dead twigs, broken earthen pots.

Delhi.

Physarina echniospora Thind \& Manocha, 1964 (Didymiaceae)

On dead wood, leaves.

Jamna Bridge, Uttar Pradesh.
Physarum aeneum (Lister) R.E. Fries., 1903

(Physaraceae)

On dead pinnae of small ferns.

Hooghly, Serampore, West Bengal.

Physarum auriscalpium Cooke, 1877

(Physaraceae)

On bark of Rhododendron sp.

Narkanda, Jakhu Hills, Simla, HImachal Pradesh; West Bengal.

Physarum bethelii Macbr., 1911 (Physaraceae)

On dead wood.

Darjeeling, West Bengal.
Physarum bitectum
G. List.,
1911(Physaraceae)
On decaying leaves of Agave sp and other angiospermic plants.
Mussoorie, Uttar Pradesh; Tamil Nadu.

Physarum bivalve Pers., 1795 (Physaraceae)

On dead leaves of Agave sp; Tectona grandis, Mangifera inidca, Ficus benghalensis, on dead leaves of Quercus incana and other plants.

Sinhagad, Pune, Karjat, Maharashtra; Dhobi ghat, Mussoorie, Uttar Pradesh; Delhi; West Bengal.

Physarum bogoriense Racib., 1898 (Physaraceae)

On fallen leaves of Ficus benghalensis, on dead and living leaves and twigs of herbaceous plants, leaves and stems of Setaria $s p$.

Pune University Campus, Pune, Maharashtra; Mussoorie, Uttar Pradesh; Seramapore, West Bengal; Cinnamara, Tocklai, Assam; Himachal Pradesh; Karnataka.

Physarum brunneolum (Phill.) Massee, 1892 (Physaraceae)

On lichens, mosses and decaying wood.

Kalatop, Dalhousie, Himachal Pradesh.

Physarum carneum G. Lister \& Sturgis, 1910 (Physaraceae)

On decaying twigs of Acacia catechu. Sagar, Madhya Pradesh.

Physarum cinereum (Batsch) Pers., 1794 (Physaraceae) 
On blades of living grasses, decaying leaves of Syzygium sp., on dead leaves and twigs often on living herbaceous plants, rarely on wood.

Pune, Sinhagad, Mahabaleshwar, Karjat, Maharashtra; Serampore, West Bengal; Mussoorie, Uttar Pradesh; Madras, Tamil Nadu; Amritsar, Punjab; Delhi; Karnataka.

Physarum citrinum Schum., 1803 (Physaraceae)

On dead leaves and angiosperm twigs.

Himachal Pradesh; Darjeeling, West Bengal; Mussoorie, Uttar Pradesh.

Physarum compressum Alb. \&. Schw., 1805 (Physaraceae)

On decaying leaves of Agave sp., Euphorbia sp., palm inflorescence, on bark of dead branches of Erythrina indica, living grass and vegetable debris, on dead bark of Rhododendron arboreum.

Mahabaleshwar, Sinhagad, Pune, Kanakeshwar, Maharashtra; Madras, Tamil Nadu; Coorg, Karnataka; Simla, Himachal Pradesh; Uttar Pradesh; Assam.

Physarum conglomeratum Rost., 1874 (Physaraceae)

On dead leaves.

Landour, Mussorie, Uttar Pradesh; Sikkim.

Physarum contextum (Pers.) Pers., 1801 (Physaraceae)

On dead leaves of Mangifera indica, Grevillea robusta, on dead leaves.

Lohagad, Pachgani, Maharashtra; Darjeeling, West Bengal.

Physarum crateriforme Petch, 1909 (Physaraceae)

On decaying leaves and bark of Azadirechta indica, Wrightia tinctoria.

Agricultural Horticultural Society, Garden, Tamil Nadu; Delhi; Mussoorie, Uttar Pradesh; Nazira, Assam.

Physarum decipiens Curt., 1848 (Physaraceae) On dead leaves of Tectona grandis, Ficus benghalensis.

Pune University Campus, Pune, Maharashtra.
Physarum dictyosporum Martin, 1962 (Physaraceae)

On dead and decaying leaves of Dalbergia sissoo.

Chandigarh, Himachal Pradesh.

Physarum diderma Rostaf., 1874 (Physaraceae)

On decaying leaves of Agave sp. and bark, twigs and leaves of angiospermic plants.

Mussoorie, Uttar Pradesh; Delhi; Himachal Pradesh.

Physarum didermoides (Pers.) Rostaf., 1874 (Physaraceae)

On dead bark of tree, decaying leaves of Agave sp.

Dehradun, Adunca Bridge, Mussoorie, Uttar Pradesh; Coorg, Karnataka; Simla, Himachal Pradesh.

Physarum dudlianum Lakhanpal \& Mukherji, 1981 (Physaraceae)

On dead twigs, leaves and fruits.

Delhi and Uttar Pradesh.

Physarum durjoylingum Lodhi, 1934

(Physaraceae)

On wood.

Darjeeling, West Bengal.

Physarum echinosporum Lister, 1899 (Physaraceae)

On dead leaves of Tectona grandis and Syzygium sp., on dead leaves of Mimusops elengi.

Sinhagad, Lohagad, Mahabaleshwar, Pune, Maharashtra; Sibpur, Baliganj, West Bengal; Jorhat, Assam; Chennai, Tamil Nadu; Dehradun, Uttar Pradesh.

Physarum famintzini Rost., 1874 (Physaraceae)

On dead and decaying leaves.

Delhi.

Physarum flavidum (Peck.) Peck., 1879 (Physaraceae)

On logs of wood and mosses in coniferous forest. 
Simla, Jakhu hills, Dalhousie, Himachal Pradesh.

Physarum gilkeyanum Gilbert, 1932 (Physaraceae)

On leaves of Polyalthia longifolia, as a contanimant in a petri dish containing Eleusine coracana, coffee litter, on bark of Azadirechta indica.

Pollibeta, Coorg, Karnataka; Delhi, Tamil Nadu.

Physarum globuliferum (Bull.) Pers, 1801 (Physaraceae)

On decaying $\operatorname{logs}$ of wood and decaying stumps.

Chamba - Sahu, Himcahal Pradesh; Patni top, Jammu and Kashmir.

Physarum gyrosum Rost., 1875 (Physaraceae)

On sheathing leaf bases of Cycas sp. and leaving grasses, on soil, fallen flowers, dead twigs, on leaves of Tagetes sp.

Pune, Kankeshwar, Maharashtra; Cinnamara, Jorhat, Tocklai, Assam; Amritsar, Punjab; Madras, Tamilnadu.

Physarum javanicum Racib., 1898 (Physaraceae)

On decaying gymnospermous and angiospermous wood.

Delhi.

Physarum javanicum Racib. var. macrosporum Lakhanpal \& Mukherji, 1981 (Physaraceae)

On wood, fruits of Sesamum orientale.

Delhi.

Physarum laevisporum Agnihothrudu, 1963 (Physaraceae)

On decaying leaves and twigs of Camellia sinensis.

Cinnamara, Assam; Hamirpur, Haretta, Himachal Pradesh.

Physarum lateritium (Berk. \& Rav) Morgan, 1896 (Physaraceae)

On dead leaves and twigs, sometimes on dead roots.

Jamna Bridge, Mussoorie, Uttar Pradesh.
Physarum leucophaeum Fries. \& Palmquist, 1818 (Physaraceae)

On dead leaves stem, decaying vegetable litter, animal refuse.

Serampore, West Bengal; Dehradun, Uttar Pradesh; Cinnamara, Tocklai, Assam.

Physarum leucopus Link, 1809 (Physaraceae) On dead leaves of Tectona grandis, on dead leaves and bark.

Karjat, Maharashtra; Cinnamara, Tocklai, Assam; Darjeeling, West Bengal; Amritsar, Punjab; Coorg, Karnataka; Sagar, Madhya Pradesh; Kalka, Haryana.

Physarum listeri Macbr., 1934 (Physaraceae)

On wood, needles and twigs of Picea smithiana.

Simla, Himachal Pradesh; North Western India.

Physarum luteo-album Lister \& G. Lister, 1904 (Physaraceae)

Host not mentioned.

North-Western India.

Physarum luteolum Peck, 1878 (Physaraceae) On decaying leaves, on decaying leaves of coffee.

Pachgani, Maharashtra; Karadibetta Estate, Hassan, Karnataka.

Physarum megalosporum Macbr., 1922 (Physaraceae)

On dead leaves of Ficus benghalensis.

Pune, Maharashtra.

Physarum melleum (Berk. \& Br.) Massee, 1892 (Physaraceae)

On dead leaves, living mosses, dead leaves of Quercus incana, living leaves of Gerbera sp.

Mussoorie, Uttar Pradesh; Dehradun, Uttar Pradesh; Calcutta, Darjeeling, West Bengal; Mandi, Haretta, Hamirpur, Himachal Pradesh; Chennai, Tamil Nadu; Guntur, Andhra Pradesh; Amritsar, Punjab.

Physarum mortonii Macbr., 1922 (Physaraceae)

On bark and living mosses.

Mahabaleshwar, Maharashtra. 
Physarum mutabile (Rostaf.) G. List., 1911 (Physaraceae)

On dead leaves of Ficus benghalenis, decaying leaves of Tectona grandis, on dead leaves and pods of Dalbergia sissoo, dead leaves of Saccharum sp.

Pune, Lohagad, Sinhagad, Maharashtra; Dehradun, Uttar Pradesh; Chandigarh, Himachal Pradesh; Chennai, Tamil Nadu.

Physarum nicaraguense Macbr., 1893 (Physaraceae)

On decaying bark, on decaying leaf base of Cycas circinalis, dead leaves of Agave sp., dead wood and bark of tree.

Pune, Katraj, Maharashtra; Madras, Tamilnadu; Dehradun, Uttar Pradesh; Cuttack, Orissa; Cinnamara, Assam. Hamirpur, Mandi, Haretta, Himachal Pradesh.

Physarum notabile Macbr., 1922 (Physaraceae)

On decaying leaves of Quercus incana. Narkanda, Simla, Himachal Pradesh.

Physarum nucleatum Rex, 1891 (Physaraceae) Physarum simile Rostaf., 1876

On dead and rotten wood stump, dead leaves of Quercus incana, leaf sheath of Musa paradisiaca, fronds of Pteris spp. Mussoorie, Uttar Pradesh; Cinnamara, Jorhat, Nazira, Assam; Sagar, Madhya Pradesh; Hamirpur, Mandi, Himachal Pradesh; Serampore, West Bengal.

Physarum nudum Macbr., 1932 (Physaraceae) On leaves of Bamboo and Ficus benghalensis, on green grass leaves, leaves and inflorescence of grass.

Pune, Maharashtra; Thatchi, Mandi, Himachal Pradesh; Nandi hills, Mysore, Karnataka.

Physarum nutans Pers., 1975 (Physaraceae)

On leaves of Cajanus cajan, decaying cotton balls, roots of lawn grass, moss growing on bark of trees and dead twigs.

Chennai, Tamil Nadu; Tocklai, Assam; Darjeeling, West Bengal; Sagar, Madhya Pradesh; Dehradun, Uttar Pradesh; Simal, Dalhousie, Himachal Pradesh.
Physarum oblatum Macbr., 1922

(Physaraceae)

On leaves of Agave americana and Syzygium sp., on tea soil.

Purandar fort, Maharashtra; Tocklai, Assam; Serampore, Calcutta, West Bengal; Simla, Himachal Pradesh.

Physarum ovisporum G. Lister, 1921 (Physaraceae)

On fallen leaves of Saccharum sp. and Bougainvillea sp.

Delhi.

Physarum pachaganiensis Nanir \& Tembhurne, 2011 (Physaraceae)

On living angiospermic leaves.

Pachgani, Satara, Maharashtra.

Physarum panhalensis Nanir \& Tembhurne, 2011 (Physaraceae)

Host not mentioned.

Panhala, Kolhapur, Maharashtra.

Physarum pedicellata (Gilbert) Dhillon, 1976 (Physaraceae)

On bark of Aesyculus indica

Manali, Himachal Pradesh.

Physarum pezizoideum (Jungh.) Pav. \& Lag., 1903

(Physaraceae)

on tree stems and fence posts.

Surajpur, Chandigarh; West Bengal.

Physarum psittacinum Ditmar, 1817 (Physaraceae)

On dead and decaying wood in a mixed forest. Banikhet, Dalhousie, Himachal Pradesh.

Physarum pulcherrimum Berk \& Curtis, 1873 (Physaraceae)

On dead stems of Andropogon sorghum.

Patna, Bihar.

Physarum pusillum (Berk. \& Curt.) G. List., 1911 (Physaraceae)

On bark, decaying leaves of Agave sp, decaying cord and paper.

Pune University Campus, Pune, Maharashtra; Adunca Bridge, Mussoorie, Uttar Pradesh; 
Nilgiris, Karnataka; Serampore, West Bengal; Delhi; Hamirpir, Himachal Pradesh. Amritsar, Punjab.

Physarum reniforme Lister, 1911
(Physaraceae)
On decaying leaves of Musa paradisiaca,
decaying bark of Albizia stipulata, decaying
wood and bark of trees, bushes infected with
Poria sp.
Jorhat, Borbhetta, Tocklai, Assam.

Physarum retisporum Martin, Thind \& Rehill, 1959 (Physaraceae)

On dead leaves, twigs and bark.

Mussoorie, Uttar Pradesh.

Physarum rigidum (Lister) Lister, 1925 (Physaraceae)

On rotting wood and on Aporpium sp., decaying wood of shade tree, bark of poles of Casuarina equisetifolia, dead leaves of Ipomea sp.

Mussoorie, Uttar Pradesh; Coorg, Karnataka; Madras, Tamil Nadu; Manali, Himachal Pradesh.

Physarum roseum Berk. \& Br., 1875 (Physaraceae)

On decaying leaves of Euphorbia sp., on wood and bark, dead twig of Enterolobium saman and gymnospermous plants.

Kankeshwar, Maharashtra; Dehradun, Uttar Pradesh; Tocklai, Assam; Narkanda, Simla, Himachal Pradesh.

Physarum serpula Morg., 1896 (Physaraceae) On fallen leaves of Syzygium sp, on grass, on decaying bark of Hibiscus rosa-sinensis, dead leaves of twigs of trees, decaying bamboo mat, dead twigs of Ipomea sp.

Pachgani, Mahabaleshwar, Maharashtra; Tocklai, Assam; Madras, Tamil Nadu.

Physarum sessile Brandza., 1921 (Physaraceae)

On dead leaves, decaying leaves of Musa paradisiaca, leaf sheath of Scitamineae, leaves and culms of a reed grass, on dead leaves of Mangifera indica.

Darjeeling, West Bengal; Tocklai, Jorhat,
Nagajanka, Assam; Hamirpur, Haretta, Himachal Pradesh.

Physarum sinuosum (Bull.) Wienm., 1829 (Physaraceae)

On dead leaves.

Mussoorie, Uttar Pradesh.

Physarum spinulosum Thind \& Sehgal, 1964 (Physaraceae)

On bark and mosses.

Senchal forest, Darjeeling, West Bengal.

Physarum spumariodes Lakhanpal \& Mukherji. 1979 (Physaraceae)

On decaying angiosperm leaves. Haretta, Himachal Pradesh.

Physarum stellatum (Massee) G. W. Martin, 1947 (Physaraceae)

On leaves of Agave americana, on dead wood, mosses and oak stump.

Kanakeshwar, Mahabaleshwar, Maharashtra; the Park, Mussoorie, Uttar Pradesh; Simla, Himachal Pradesh.

Physarum straminipes A. Lister, 1898 (Physaraceae)

On dry petiole of Phoenix sp. in moist chamber culture.

Ahmadpur, Latur, Maharashtra.

Physarum sulphureum Alb. \& Schw., 1805 (Physaraceae)

On dead decaying angiospermic leaves.

North Point, Darjeeling, West Bengal; Coorg, Polibetta, Karnataka, Mussoorie, Uttar Pradesh; Tocklai, Cinnamara, Jorhat, Assam.

Physarum sumatii Patil \& Ranade 1975 (Physaraceae)

On dead and decaying leaves.

Kanakeshwar, Maharashtra.

Physarum superbum Hagelst., 1940 (Physaraceae)

On Phylloclades of Euphorbia sp., on dead leaves.

Kanakeshwar, Maharashtra; Darjeeling, West Bengal; Mussoorie, Uttar Pradesh. 
Physarum tenerum Rex, 1890 (Physaraceae)

On dead leaves of Tectona grandis, Syzygium sp., Mangifera indica, Ficus benghalensis, on dead wood.

Katraj, Sinhagad, Khandala, Lohagad, Pune, Karjat, Amboli, Pachgani, Maharashtra; Mussoorie, Uttar Pradesh; Simla, Himachal Pradesh; Kodaikanal, Tamil Nadu.

Physarum thindii Patil \& Ranade 1975 (Physaraceae)

On decaying leaves of Memeceylon umbelatum.

Mahabaleshwar, Maharashtra.

Physarum urnae Singh \& Pusphavathy, 1979

(Physaraceae)

On living grass leaves.

Delhi.

Physarum variegatum Thind \& Dhillon, 1967

(Physaraceae)

On decaying stump.

Darjeeling, West Bengal.

Physarum vernum Somm ex Fr., 1829 (Physaraceae)

On fallen leaves of Tectona grandis and Syzygium sp., on decaying plant material, dead leaves of Agave sp., living leaves of Ipomea sp., bark of dead branches.

Mahabaleshwar, Lohagad, Karjat, Pune, Maharashtra; Madras, Tamil Nadu; Mussorie, Uttar Pradesh; Delhi; Coorg, Karnataka; Amritsar, Punjab; Mandi, Haretta, Hamirpur, Himachal Pradesh.

Physarum virescens Ditmar, 1817 (Physaraceae)

On dead leaves and twigs, cladodes of Parkinsonia aculeata.

Darjeeling, West Bengal; Chennai, Tamil Nadu.

Physarum viride (Bull.) Pers., 1795 (Physaraceae)

On wood, on rotten wooden stump.

Lohagad, Maharashtra; Kempty Falls, Mussoorie, Uttar Pradesh; Delhi, Benjar, Himachal Pradesh; Darjeeling, West Bengal.

Physarum viride var. aurantium (Bull) Lister, 1894 (Physaraceae)
On dead wood in Oak forest.

Chamba; Khijjiar, Dalhousie, Himachal Pradesh; Mussoorie, Uttar Pradesh.

Physarum viride var. hinnuleum G. Lister, 1924 (Physaraceae)

On decayed wood.

Tindharia, West Bengal; Uttar Pradesh.

Pleiomorpha belmontiana (Nann.-Brem.)

Dhillon, 1978 (Liceaceae)

On bark of Abies sp.

Manali, Himachal Pradesh.

Pleiomorpha biforis (Morgan) Dhillon, 1980 (Liceaceae)

On bark of Populus sp.

Narkanda, Himachal Pradesh.

Pleiomorpha pedicellata (Gilbert) Dhillon, 1978 (Liceaceae)

On bark of Aesculus indica.

Manali, Himachal Pradesh.

Reticularia jurana Meylan, 1908

(Reticulariaceae)

On bark.

Manali, Himachal Pradesh.

Reticularia lycoperdon Bull., 1790 (Reticulariaceae)

On bark of Pinus excelsa, soil between tea bushes.

Dalhousie, Mandi, Himachal Pradesh; Cinnamara, Jorhat, Tocklai, Assam.

Reticularia splendens Morgan, 1893 (Reticulariaceae)

On bark.

Chandigarh, Himachal Pradesh.

Reticularia venulosa Berk. \& Curt., 1868 (Reticulariaceae)

On dead wood.

Khandala, Bombay, Maharashtra.

Stemonitis axifera (Bull.) Macbr., 1889 (Stemonitaceae)

On wood, on dead wood, living green mosses, decaying tea frames, on decaying coniferous wood.

Mahabaleshwar, Maharashtra; Tindharia, 
Darjeeling, West Bengal; Mussoorie, Uttar Pradesh; Nazira, Tocklai, Assam; Mandi, Simla, Narkanda, Himachal Pradesh.

Stemonitis confluens Cooke \& Ellis, 1876 (Stemonitaceae)

On wood of dead stump.

Polibetta, Coorg, Karnataka; Chennai, Tamil Nadu.

Stemonitis farrensis Lakhanpal \& Mukherji, 1977 (Stemonitaceae)

On decayed gymnosperm wood covered with mosses.

Narkanda, Simla, Himachal Pradesh.

Stemonitis flavogenita Jahn., 1904 (Stemonitaceae)

On dead rotting wood, dead archegoniosphore of Marchantia sp.

Cuttack, Orissa; Darjeeling, West Bengal; Thatchi, Mandi, Simla, Himachal Pradesh; Delhi University Campus, Delhi.

Stemonitis fusca Roth., 1787 (Stemonitaceae) On decaying wood, on dead gymnosperm wood.

Mahabaleshwar, Kanakeshwar, Matheran, Maharashtra; Jorhat, Assam; Dalhousie, Narkanda, Hamirpur, Nainital, China Peak, Himachal Pradesh; Mussoorie, Uttar Pradesh; Coorg, Karnataka; Darjeeling, West Bengal; Cuttack, Orissa; Chennai, Tamil Nadu.

Stemonitis fusca Roth. var. papillosa Meylan., 1935 (Stemonitaceae)

On decaying wood.

Thatchi, Mandi, Himachal Pradesh.

Stemonitis graciliformis Nann.-Brem., K.G. Mukerji \& R. Pasricha, 1984 (Stemonitaceae) On herbaceous stem.

Sagar, Madhya Pradesh.

Stemonitis herbatica Peck, 1873 (Stemonitaceae)

On living grasses, decaying leaves, on sandy garden soil, stem of Ipomea sp., Maize leaf, fern fronds, living and dead angiospermic leaves.
Pune, Vavoshi, Maharashtra; Cinnamara, Nazira, Assam; Delhi, Chennai, Tamil Nadu; Dehradun, Uttar Pradesh; Hamirpur, Himachal Pradesh; Amritsar, Punjab; Darjeeling, Serampore, West Bengal.

Stemonitis inconspicua Nann.-Brem., 1966 (Stemonitaceae)

On decaying coniferous wood.

Thatchi, Mandi, Himachal Pradesh.

Stemonitis mussooriensis Martin, Thind \& Sohi, 1957 (Stemonitaceae)

On decayed bark.

Jamna Bridge, Mussoorie, Uttar Pradesh.

Stemonitis nigrescens Rex, 1891 (Stemonitaceae)

On bark of decaying logs, decaying twigs, leaves and stem of grass, on decaying gymnospermous wood.

Fruit Research Station, Borliar, Tamil Nadu; Jorhat, Tocklai, Assam; Dalhousie, Simla, Himachal Pradesh; Pune, Maharashtra; Karnataka; Sagar, Madhya Pradesh.

Stemonitis pallida Wingate, 1899 (Stemonitaceae)

On dead wood, dead moist log.

Mussoorie, Dehradun, Uttar Pradesh; Coorg, Karnataka; Dalhousie, Himachal Pradesh.

Stemonitis smithii Macbr., 1893 (Stemonitaceae)

On rotten fire wood, stump of Cryptomeria japonica, on moist log, on mosses and bark.

Cuttack, Orissa; Darjeeling, West Bengal; Coorg, Karnataka; Simla, Himachal Pradesh; Assam.

Stemonitis splendens $\quad$ Rost., 1874 (Stemonitaceae)

On dead wood, decaying bark and leaves of Mangifera indica.

Cuttack, Orissa; Doiwala, Mussoorie, Uttar Pradesh; Delhi; Mandi, Dalhousie, Himachal Pradesh; Cinnamara, Jorhat, Tocklai, Assam; Coorg, Karnataka; Sagar, Madhya Pradesh; Darjeeling, West Bengal. 
Stemonitis travancorensis Erady, 1953 (Stemonitaceae)

On Cereus sp.

Travancore, Kerala.

Stemonitis uvifera T. Macbr., 1922 (Stemonitaceae)

On dead wood, on tea pruning litter.

Dalhousie, Himachal Pradesh; Cinnamara, Tocklai, Assam.

Stemonitis virginiensis $\quad$ Rex, 1891 (Stemonitaceae)

On decaying wood, decaying frames of tea bushes.

Cinnamara, Jorhat, Tocklai, Assam.

Stemonitis webberi Rex, 1891 (Stemonitaceae) On dead wood.

Bhillaru, Khad, Mussoorie, Uttar Pradesh; Cinnamara, Tocklai, Assam; Kalka, Punjab; Darjeeling, West Bengal; Kulu, Himachal Pradesh.

Stemonaria reticulospora Nann-Brem., R. Sharma \& K.S. Thind, 1984 (Stemonitaceae)

On burnt stump of Cryptomeria sp.

West Bengal.

Stemonitopsis hyperopta (Meylan) Nann.Brem., 1975 (Stemonitaceae)

On decaying gymnosperm wood.

Himachal Pradesh; Uttar Pradesh.

Stemonitopsis irregularis (Rex) Nann.-Brem., 1981 (Stemonitaceae)

On decayed conifer wood.

Simla, Himachal Pradesh; Mussoorie, Uttar Pradesh.

Stemonitopsis suksdorfii (Ellis \& Ev.) Nann.Brem., 1981 (Stemonitaceae)

On bark of Murraya exotica.

Coorg, Karnataka.

Stemonitopsis typhoides (Bull.) Nann - Brem., 1981 (Stemonitaceae)

On decaying gymnosperm and angiosperm wood.

Cuttack, Orissa; Jorhat, Assam; Dalhousie, Mandi, Simla, Himachal Pradesh; Uttar
Pradesh; Darjeeling, West Bengal and Southern India.

Symphytocarpus herbaticus Ing. \& Nann.Brem., 1967

On petioles and fronds of fern.

Mandi, Hamirpur, Haretta, Himachal Pradesh.

Trichia affinis de Bary, 1869 (Trichiaceae)

On dead wood, bark and wood of a stump and alive mosses growing on it.

Kotgarh, Simla hills, Himachal Pradesh; Darjeeling, West Bengal; Coffee Research Campus, Balehonoor, Karnataka.

Trichia botrytis (J. F. Gmel.) Pers., 1794 (Trichiaceae)

On bark of tree and mosses growing on it, on dead coniferous wood and living mosses.

Darjeeling, West Bengal; Dalhousie, Himachal Pradesh.

Trichia crateriforme Martin, 1963 (Trichiaceae)

On decaying gymnosperm wood.

Dalhousie, Himachal Pradesh.

Trichia drakii Lodhi, year not given (Trichiaceae)

On dead and decaying leaves.

Darjeeling, West Bengal.

Trichia decipiens (Pers.) Macbr., 1899 (Trichiaceae)

On dead and decaying wood.

Simla, Manali, Kulu, Nainital, Dalhousie, Himachal Pradesh; Mussoorie, Uttar Pradesh; Darjeeling, West Bengal.

Trichia favoginea (Batsch.) Pers., 1794 (Trichiaceae)

On decaying leaves of Agave sp., on rotten wood, on dead gymnospermous wood, bark and mosses.

Lingmala, Mahabaleshwar, Maharashtra; North Western India; Mussoorie, Uttar Pradesh; Darjeeling, West Bengal; Kumarain, Narkanda, Kulu, Simla, Himachal Pradesh; Southern India.

Trichia floriformis (Schw.) G. List., 1919 
(Trichiaceae)

On leaves and woods, live mosses, on dead coniferous wood.

Dalhousie, Narkanda, Manali, Kulu, Himachal Pradesh; Darjeeling, West Bengal.

Trichia lutescens (Lister.) Lister, 1897

(Trichiaceae)

On dead decaying wood.

Mussoorie, Uttar Pradesh.

Trichia operculata Bruhl. \& Sen-Gupta, 1927 (Trichiaceae)

On decaying leaves of palm petioles.

Baliganj, Calcutta, West Bengal.

Trichia persimilis P. $\quad$ Karst., 1868 (Trichiaceae)

On dead and decaying coniferous bark and wood.

Kotgarh, Simla Hills, Himachal Pradesh.

Trichia pusilla (Hedw.) Martin, 1949 (Trichiaceae)

On dead wood.

Darjeeling, West Bengal; Mussorie, Uttar Pradesh.

Trichia scabra Rostaf., 1875 (Trichiaceae)

On dead rotten gymnospermous wood.

The Park, Mussoorie, Uttar Pradesh; Thatchi, Mandi, Simla, Kulu, Narkanda, Himachal Pradesh.

Trichia subfusca Rex, 1890 (Trichiaceae)

On dead wood, on decaying gymnospermous wood.

Sarahan, Mahasu, Kulu, Manali, Himachal Pradesh.

Trichia subretisporum Lakhanpal \& Mukerji, 1980 (Trichiaceae)

On decaying gymnospermous wood.

Mandi, Thatchi, Manali, Himachal Pradesh.

Trichia varia (Pers.) Pers., 1800 (Trichiaceae)

On rotten and decaying gymnospermous wood.

Bhimtal, Dehradun, Mussoorie, Uttar Pradesh;

Simla, Kulu, Narkanda, Himachal Pradesh.

Trichia verrucosa Berk., 1860 (Trichiaceae)
On decaying gymnospermous wood.

Narkanda, Simla, Dalhousie, Himachal Pradesh.

Tubifera ferruginosa (Batsch.) J.F. Gmel., 1792 (Reticulariaceae)

On dead wood, on dead stump of Quercus incana; on dead gymnospermous wood and conifers.

Kotgarh, Simla, Kulu, Narkanda, Himachal Pradesh; Mussoorie, Uttar Pradesh; Darjeeling, West Bengal.

Tubifera microsperma (Berk. \& Curt.) Martin, 1947 (Reticulariaceae)

On dead decaying wood of Quercus incana; on dead stump of Cocos nucifera.

Mussoorie, Uttar Pradesh; Darjeeling, West Bengal; Chennai, Tamil Nadu.

Tubifera papillata Martin, Thind \& Sohi, 1957 (Reticulariaceae)

On rotten wood.

Mussoorie, Uttar Pradesh; Simla, Himachal Pradesh.

\section{Acknowlegments}

We are thankful Dr. (Mrs.) P. K. Jite, Mycology Laboratory, Department of Botany, University of Pune for constant encouragement.

\section{References}

Agnihothrudu V, Chinnappa CC 1966 - Some slime molds of Southern India - VII. Sydowia, 20, 183-185.

Agnihothrudu V, Chinnappa CC 1969 - Some slime molds of Southern India - VI. Mycopathologia., 38(4), 363-366.

Agnihothrudu V 1952 - Some slime molds from Southern India - I. Journal of the Indian Botanical Society, 33, 171-181

Agnihothrudu V 1954a, b - Some slime moulds of Southern India I and II. Journal of Indian Botanical Society, 33, $177-181,182-188$.

Agnihothrudu V 1955 - Some slime moulds of Southern India III. Journal of Indian Botanical Society, 34, 85-97. 
Agnihothrudu V 1956a, b - Some slime moulds of Southern India IV and V. Journal of Indian Botanical Society, 35, $27-37,210-221$.

Agnihothrudu V 1956 - An undescribed Myxomycetes from the coffee estated of Mysore - Licea mysorensis. Indian Phytopathology, 18, 92-94.

Agnihothrudu V 1958 - Notes on fungi from North East India - II. An undescribed Myxomycetes from Assam. Journal of the Indian Botanical Society, 37, 499503.

Agnihothrudu V 1959 - Notes of Fungi from North East India IV. Journal of Indian Botanical Society, 38, 418-491.

Agnihothrudu V 1961 - A List of Indian Myxomycetes. Journal of Madras University, B. 1-20.

Agnihothrudu V 1963 - Physarum laevisporum. Sydowia, 16, 121.

Agnihothrudu V 1968 - Some slime moulds of Southern India - 10. Sydowia, 22, 179 -182 .

Ainsworth CG, Susman AF 1973 - The Fungi "An advance treatise". IVB, xxii+504pp, Academic press, London.

Alexopoulos CJ 1963 - The Myxomycetes. Botanical Review, 29, 1-78.

Alexopoulos CJ 1967 - Taxonomic studies in the Myxomycetes - I. The genus Macbrideola. Mycologia, 59, 103-116.

Alexopoulos CJ 1969 - The experimental approach to the taxonomy of Myxomycetes. Mycologia, 61, 219239.

Alexopoulos CJ, Mims CW \& Blackwell M 1996 - Introductory Mycology, $4^{\text {th }}$ Edition, XVI + 613 pp. John, Wiely \& Sons. Inc. New York \& London.

Berkeley MJ 1839 - Notices on British Fungi. Ann. Nat. Hist. Ser. I, vol 1.

Bhide VP, Sathe AV, Pande Alaka, Patwardhan PG, Rao VG 1987 - Fungi of Maharashtra Supplement I, Maharashtra Association for the Cultivation of Science, Pune - 411004, $1-2$.

Bilgrami KS, Jamaluddin S, Rizwi MA 1979 Fungi of India Part - I, List and References. Today \& Tomorrow's
Printers and Publishers, New Delhi $110005,1-467$.

Bisby GR 1914 - Some observations on the formation of capillitium and the development of Physarella mirabilis and Stemonitis fusca Roth. American Journal of Botany, 1, 274-288.

Bruhl P, Gupta JS 1927 - Indian Slime Fungi (Myxomycetes or Mycetozoa). First contribution. Journal Department of Science, University of Calcutta, 8, 101122.

Butler EJ, Bisby GR 1931 - The Fungi of India. Imperial Council of Agriculture Research, India Science Monograph, 1. Calcutta, 1-237.

Butler EJ, Bisby GR revised by RS Vasudeva 1960 - The Fungi of India, Indian Council of Agricultural Research, New Delhi, 1-552.

Chavan PD, Kulkarni UV 1974 - Additions to the fungi from Maharashtra, India. Maharashtra Vidyan Mandir Patrika 9, 132-139

Dhillon SS 1976 - Myxomycetes of North Western Himalayas. Ph. D. Thesis, Punjab University, Chandigarh.

Dhillon SS, Nannenga - Bremekamp NE 1977 - Notes on some Myxomycetes from North - Western part of the Himalaya. K. Ned. Akad. Wet. Proc. C, 80, 257266.

Dhillon SS, Nannenga - Bremekamp NE 1978 - Notes on some Myxomycetes from North - Western part of the Himalaya. K. Ned. Akad. Wet. Proc. C, 81, 141149.

Dhillon SS 1977 - Myxomycetes New to India - I. Sydowia 30, 1-5.

Dhillon SS 1977 - Myxomycetes New to India - II. Sydowia 31, 27-29.

Farr ML 1962 - Arcyria cinerea and Arcyria pomiformis, revised. Mycologia, 54, 516-520.

Ghosh GR, Datta BG 1962a - Myxomycetes from Orissa (India) - I. Mycopathologia., 16, 165-176.

Ghosh GR, Datta BG 1962b - Myxomycetes from Orissa (India) - II. Mycopathologia., 16, 209-218.

Ghosh GR, Datta BG 1962c - Myxomycetes 
from Orissa. Proceeding $49^{\text {th }}$ Indian Science Congress, Part III, 243.

Ghosh GR, Datta BG 1963 - Myxomycetes from Orissa (India) - III. Mycopathologia., 19, 271-282.

Hosagoudar VB, Abraham TK, Pusphangadan P 1996 - Fungi of Kerala. Tropical Botanic Garden and Research Institute, $1-151$.

Indira PU 1968a - Some slime molds from Southern India - VIII. Journal of Indian Botanical Society, 47, 155-186.

Indira PU 1968b - Some slime molds from Southern India - IX. Distribution, habitat and Variation. Journal of Indian Botanical Society, 47, 330-340.

Indira PU 1975 - Some slime molds of Southern India - XI. Kavaka, 3, 41-54.

Jagtap AP, Singh NP 2002 - Biodiversity of The Western Ghats of Maharashtra Current Knowledge. Published by Bishen Singh Mahendra Pal Singh, Dehradun, Appendix XXV, 339-342.

Jamaluddin S, Goswami MG, Ojha BM 2004 Fungi of India 1989 - 2001. Scientific Publishers (India), Jodhpur, 1-326.

Kamat MN, Patwardhan PG, Rao VG, Sathe AV 1971 - Fungi of Maharashtra, Bulletin No. 1. Maharashtra Association for Cultivation of Science, Poona \& Mahatma Phule Krishi Vidyapeth, Rahuri, Maharashtra, 1124.

Kar A 1964 - Myxomycetes of Calcutta Suburbs. Indian Phytopathology 17, 222-223.

Kaur S, Mukherji KG 1996 - Arcyria rufosa: A new Indian Myxomycetes. Mycotaxon, $59,479-481$.

Kowalski DT, Lakhanpal TN 1973 - A new species of Didymium from India. Mycologia, 65, 474-477.

Lakhanpal TN 1966 - Taxonomic studies on the Mxyomycetes of Simla and Kulu Hills (Himachal Pradesh). M. Sc. Thesis, Punjab University, Chandigarh.

Lakhanpal TN 1978 - Taxonomic studies on the Indian Myxomycetes - XVIII. Two species of Myxomycetes and a reappraisal of the genus Lepidodermopsis. Norwegian Journal of Botany, 25, 196.

Lakhanpal TN, Mukherji KG 1981 Taxonomy of the Indian Myxomycetes. J. Cramer, FL - 9490 VADUZ, 01-411. Lister G 1924 - Mycetozoa from North India. Journal of Botany, 62, 16-20.

Lister G 1925 - A monograph of Mycetozoa. $03^{\text {rd }}$ Edition, British Museum (Nat. Hist.) London, 296.

Lodhi SA 1934 - Indian slime molds (Myxomycetes) (Being Descriptions of the species collected by Late Mrs. Drake) University of Punjab, Lahore, reprinted by: Sushma Publications 318 A, Dehradun (1981), 1-34, Plate I XVII.

Martin GW 1932 - Systematic Position of Myxomycetes and its bearing on Classification of Fungi. Botanical Gazzette, 93, 421-435.

Martin GW, Thind KS, Sohi HS 1957 - The Myxomycetes of Mussorie Hills - IV. Mycologia, 49, 128-133.

Martin GW, Alexopoulos CJ 1969 - The Myxomycetes. University of Iowa Press, Iowa, pp 560.

Mathur RS 1964 - Myxomycetes of India. Lab. Dev. Jour. Sci. Tech. 2, 164 - 174.

Mishra RL, Ranade VD 1979 - Mxyomycetes of Maharashtra - II. The genus Diderma. Maharashtra Vidnyan Mandir Patrika, 14, 33-41.

Mukherji KG, Juneja RC 1975 - Fungi of India Supplement to the list of Fungi - 1962 - 1972. Emkay Publications Delhi, 1250.

Mundkur BB 1938 - Fungi of India. Supplement - I. The Imperial Council of Agricultural Research. Science Monograph, 12, 1-54.

Nanir SP 1979 - Myxomycetes of Marathwada. Ph.D thesis, Marathwada University.

Nanir SP 1985 - Contribution to the knowledge of Myxomycetes from India - III B. Indian Botanical Reporter - IV (1), 42-45.

Nanir SP, Rokade BG 1987 - Myxomycetes of Marathwada - I. Marathwada University Journal of Science, 12.

Nannega - Bremekamp NE 1958a - Notes on 
Myxomycetes. I. Some remarks on the genus Reticularia. Bull. Emend. Rost. Med. Bot. Mus. Herb. Utrecht. 149, 769-779.

Nannega - Bremekamp NE 1958b - Notes on Myxomycetes. II. Med. Bot. Mus. Herb. Utrecht., 150, 780-785.

Nannega - Bremekamp NE 1967 - Notes on Myxomycetes. XII. A revision of Stemonitales. K. Nad. Acad. Wat. Proc. 70, 201-216.

Pathak NC, Ghosh RN 1962 - Fungi of Uttar Pradesh. Bulletin National Botanical Gardens, Lucknow.

Patel MK, Kamat MN , Bhide VP 1948 - The Fungi of Bombay Supplement I. Indian Phytopath. 2, 142-155

Patil SD, Mishra RL 1977 - An atypical form of Physarum pusillum. Maharashtra Vidnyan Mandir Patrika, 12 (1), 41-42.

Patil SD, Ranade VD 1975 - Myxomycetes of Maharashtra - I. The genus Diderma. Pers. Indian Science Congress (Abstracts). 62 ${ }^{\text {nd }}$ Session.

Patwardhan PG, Joshi GT 1975 Myxomycetes of Maharashtra, India. Part - I. Bio - Vigyanam, 1, 49 - 53.

Ramakrishnan K, Subramanian CV 1952 - The Fungi of India - A Second Supplement. Journal of Madras University, B. 22, page no. $1-65,163-182$.

Ranade VD, Mishra RL 1977 - Myxomycetes of Maharashtra - III. Maharashtra Vidnyan Mandir Patrika, 12: 25 - 27.

Ranade VD 1978 - Myxomycetes of Maharashtra, Ph.D Thesis, University of Pune, 1-226.

Rangaswamy G, Sheshadri VS, Lucy Channama KA 1970 - Fungi of South India. International Book House Private Limited, Bombay.

Rokade BG, Nanir SP 1993 - Myxomycetes of Jalgaon and Dhule District (Khandesh): India. Abstract N. C. R. A. P, 14.

Roy TC 1959 - Fungi of Bengal. Bull. Bot. Soc. Bengal, Calcutta.

Sarbhoy AK, Lal G, Varshney JL 1975 - Fungi of India (1967 - 71). Navyug Traders, New Delhi.

Sarabhoy AK, Agarwal DK, Varshney J. L. (1980) - Fungi of India - 1971 - 1976.
Navyug Traders Booksellers \& Publishers, New Delhi, 1-277.

Sarabhoy AK, Agarwal DK, Varshney JL 1986 - Fungi of India - 1977 - 1981. Associated Publishing Company, New Delhi, 1-274.

Sekhon SS 1976 - The genus Licea Schrad. in India. Proc. $63^{\text {rd }}$ Indian Sci. Conference. Part III, 60.

Sekhon SS 1978 - The Myxomycetes of Chandigarh - I. Journal of Indian Botanical Society, 57, $331-337$.

Sekhon SS 1979a - The Myxomycetes of Chandigarh - II. Journal of Indian Botanical Society, 58, 16-20.

Sekhon SS 1979b - The genus Badhamia Berk. in India. Journal of Indian Botanical Society, 58, 56-83.

Singh HH, Pushpavathy KK 1965 - The Slime Molds of Delhi - I Mycopathologia., 27, 23-40.

Singh HH, Pushpavathy KK 1966 - The Slime Molds of Delhi - II Mycopathologia., 28, 265-272.

Singh H, Pushpavathy KK, Sethi I 1979 - The Slime Molds of Delhi - III. Proceeding of Indian National Science Academy, 45, 375-382.

Sood R, Lakhanpal TN 1980 - Taxonomic studies of Indian Myxomycetes - XIX. Sydowia, Vol. XXXIII, 174-180.

Subramaniam CV, Ramakrishnan K 1956 List of Indian Fungi - 1952 - 1956. Journal of Madras University B. 26, $327-421$.

Subramaniam CV, Tyagi PD 1964 - List of Indian Fungi -1956 - 1960. Journal Madras University, 34, 1-134.

Tandon RN, Chandra S 1963/64 - Supplement to the list of Indian Fungi $1957-62$. University of Allahabad studies (Botany Publication); PUBL University Allahabad, Allahabad.

Tembhurne RR, Nanir SP 2011 - The Myxomycetes of South - West Maharashtra (India) - III. Plant Science Feed, 1 (2), 23-27.

Tembhurne RR, Nanir SP 2011 - New five species of the Myomycetes recorded from the South - East Region of Maharashtra (India). Science Reporter 1 
(2), 65-68.

Thind KS, Rehill PS 1957 - The Myxomycetes of the Mussoorie Hills - VII. Indian Phytopathology 10, 85-96.

Thind KS, Sehgal HS 1960 - The Myxomycetes of India - XIII. Indian Phytopathology 13, 103-117.

Thind KS, Manocha MS 1963 - The Mxyomycetes of India - XV. Indian Phytopathology 16, 177-184.

Thind KS, Sehgal HS 1964 - The Myxomycetes of India - XVI. Mycologia 56, 561-567.

Thind KS, Khara HS 1969 - The Myxomycetes of India - XXIII. Proceedings of Indian Academy of Science, 69, 284-293

Thind KS 1977 - The Myxomycetes of India. Indian Council of Agricultural Research, New Delhi, page no. 1-452.
Thite AN 1975 - New and Noteworthy Fungi from Maharashtra. Maharashtra Vidnyan Mandir Patrika, 9, 117-120.

Tilak ST, Rao R 1968 - Second Supplement to the Fungi of India - 1962 - 1967, Marathwada University, Marathwada, 1 -312 .

Uppal BN, Patel MK, Kamat MN 1935 - The Fungi of Bombay, Bulletin number 176 of 1934, Department of Agriculture, Bombay. Printed at the Government Central Press, VIII, 1-56.

Vasudeva RS 1962 - Fungi of India Supplement - I. Indian Council of Agricultural Research, New Delhi, 1206.

Wrigley D, Lado C 2005 - A taxonomic evaluation of the stipitate Licea species. Fungal Diversity 20, 261-314. 